\title{
COLA. III. RADIO DETECTION OF ACTIVE GALACTIC NUCLEUS IN COMPACT MODERATE LUMINOSITY INFRARED GALAXIES
}

\author{
R. Parra ${ }^{1,6}$, J. E. Conway ${ }^{1}$, S. Aalto ${ }^{1}$, P. N. Appleton ${ }^{2}$, R. P. Norris 33 , Y. M. Pihlström ${ }^{4,7}$, and L. J. Kewley \\ 1 Onsala Space Observatory, SE-439 92 Onsala, Sweden \\ ${ }^{2}$ NASA Herschel Science Center, Mail Code 100-22, California Institute of Technology. 770 S. Wilson Blvd., Pasadena, CA 91125, USA \\ ${ }^{3}$ CSIRO Australia Telescope National Facility, P.O. Box 76, Epping, NSW 1710, Australia \\ ${ }^{4}$ Department of Physics and Astronomy, UNM, 800 Yale Blvd., NE, Albuquerque, NM 87131, USA \\ ${ }^{5}$ University of Hawaii, 2680 Woodlawn Drive, Honolulu, HI 96822, USA \\ Received 2009 July 31; accepted 2010 June 13; published 2010 August 12
}

\begin{abstract}
We present results from 4.8 GHz Very Large Array (VLA) and global very long baseline interferometry (VLBI) observations of the northern half of the moderate FIR luminosity (median $L_{\mathrm{IR}}=10^{11.01} L_{\odot}$ ) COLA sample of star-forming galaxies. VLBI sources are detected in a high fraction (20/90) of the galaxies observed. The radio luminosities of these cores $\left(\sim 10^{21} \mathrm{~W} \mathrm{~Hz}^{-1}\right)$ are too large to be explained by radio supernovae or supernova remnants and we argue that they are instead powered by active galactic nuclei (AGNs). These sub-parsec scale radio cores are preferentially detected toward galaxies whose VLA maps show bright 100-500 parsec scale nuclear radio components. Since these latter structures tightly follow the FIR to radio-continuum correlation for star formation, we conclude that the AGN-powered VLBI sources are associated with compact nuclear starburst environments. The implications for possible starburst-AGN connections are discussed. The detected VLBI sources have a relatively narrow range of radio luminosity consistent with models in which intense compact Eddingtonlimited starbursts regulate the gas supply onto a central supermassive black hole. The high incidence of AGN radio cores in compact starbursts suggests little or no delay between the starburst phase and the onset of AGN activity.
\end{abstract}

Key words: galaxies: active - galaxies: starburst - infrared: galaxies - radio continuum: galaxies

Online-only material: extended figure, machine-readable tables

\section{INTRODUCTION}

It seems likely that supermassive black holes (SMBHs) are ubiquitous in galactic nuclei. The nuclear stellar-velocity dispersion and bulge-size to black hole mass correlations (Magorrian et al. 1998; Merloni et al. 2003; Häring \& Rix 2004) indicate that most galaxies with central bulges should contain SMBHs at their centers. The presence of a SMBH is a necessary condition for active galactic nucleus (AGN) activity but only a small fraction of galaxies at any given time are strong AGN sources, implying that a decisive factor determining AGN luminosity is the gas feeding mechanism. While for Seyfert or LINER class AGNs only relatively small black hole accretion rates are required $\left(0.01\right.$ to $0.1 M_{\odot}$ year $\left.^{-1}\right)$, gas originating in the galactic disk must lose angular momentum in order to be accreted onto the central black hole. Various mechanisms to achieve such momentum transfer and mass transport have been suggested (see review by Knapen 2004).

There is increasing observational evidence for an AGN-starburst connection (Davies et al. 2007; Heckman 2008; Prieto et al. 2005, 2007; Fathi et al. 2006). Plausibly, such circumnuclear starbursts may play a role in removing angular momentum and so feeding the central black hole (Schartmann et al. 2010). Alternatively, both nuclear starbursts and AGNs could be independent consequences of a common gas transport mechanism into the centers of galaxies with no direct causal connection between the two phenomena. Understanding in detail the phenomenology of starburst and AGN activity, in particular whether

\footnotetext{
6 Now at European Southern Observatory, Alonso de Cordova 3107, Casilla 19001, Santiago 19, Chile.

7 Also Adjunct Astronomer at the National Radio Astronomy Observatory.
}

one phase begins before the other, is important to test the nature of the starburst-AGN inter-relationship.

Most detailed observational work so far on the starburst-AGN connection has studied starburst activity in samples of AGNs (Davies et al. 2007). Here we adopt the alternative approach of searching for AGN activity in a far infrared-selected sample of star-forming galaxies known as Compact Objects in Low-power AGNs (COLA; Corbett et al. 2002, 2003, see Section 2 for sample definition). Our principal means of identifying AGN activity in this sample is searching for high brightness temperature very long baseline interferometry (VLBI) radio cores. Classic optical emission line diagnostics (Baldwin et al. 1981; Veilleux \& Osterbrock 1987; Kewley et al. 2001; Kauffmann et al. 2003) can be unreliable for such searches as an AGN may be obscured by dust in star-forming galaxies. High brightness-temperature radio emission is unambiguous evidence of an AGN, however a non-detection does not exclude an AGN because of the existence of both radio-loud and radio-quiet objects (see Zamfir et al. 2008, for a recent review); it follows that VLBI searches can only give a lower limit to the AGN fraction. It should be noted that while radio supernovae ( $\mathrm{SNe}$ ) can also generate compact radio emission they have a maximum luminosity which distinguishes them from AGNs (see Corbett et al. 2002, and Section 5.1.4).

We have already published radio observations (Corbett et al. 2002) and optical spectroscopy results (Corbett et al. 2003) for the $\delta<0^{\circ}$ (COLA-S) part of the sample. Here we present radio and optical observations of the northern half $\left(\delta>0^{\circ}\right)$ of the sample (COLA-N). This paper is organized as follows. In Section 2, we give an overview of the COLA project and previous observations. In Section 3, we describe our Very Large Array (VLA), VLBI, optical spectroscopy and other observations and give an overview of the data reduction process. 
Table 1

COLA North Sample

\begin{tabular}{|c|c|c|c|c|c|c|c|c|c|}
\hline $\begin{array}{l}I R A S \\
\text { Name } \\
(1)\end{array}$ & $\begin{array}{l}\text { Other } \\
\text { Name } \\
(2)\end{array}$ & $\begin{array}{l}\text { R.A. } \\
\text { J2000 } \\
\text { (3) }\end{array}$ & $\begin{array}{c}\text { decl. } \\
\text { J2000 } \\
(4)\end{array}$ & $\begin{array}{c}V_{\mathrm{Hel}} \\
\left(\mathrm{km} \mathrm{s}^{-1}\right) \\
(5)\end{array}$ & $\begin{array}{c}D_{L} \\
(\mathrm{Mpc}) \\
(6)\end{array}$ & $\begin{array}{c}\log L_{\mathrm{IR}} \\
\left(L_{\odot}\right) \\
(7)\end{array}$ & $\begin{array}{c}\log L_{1.4} \\
\left(\mathrm{~W} \mathrm{~Hz}{ }^{-1}\right) \\
(8)\end{array}$ & $\begin{array}{c}\log L_{\mathrm{FIR}} \\
\left(\mathrm{W} \mathrm{Hz}^{-1}\right) \\
(9)\end{array}$ & $\begin{array}{l}q_{1.4} \\
(10)\end{array}$ \\
\hline $00005+2140$ & MRK334 & 000309.74 & 215736.46 & 6579 & 93.4 & 11.07 & 22.46 & 24.74 & 2.27 \\
\hline $00073+2538$ & N23 & 000953.58 & 255526.40 & 4566 & 64.7 & 11.09 & 22.57 & 24.82 & 2.25 \\
\hline $00506+7248$ & & 005404.00 & 730511.70 & 4706 & 69.3 & 11.49 & 22.82 & 25.21 & 2.40 \\
\hline $00521+2858$ & & 005450.27 & 291447.60 & 4629 & 65.2 & 10.88 & 22.39 & 24.62 & 2.23 \\
\hline $00548+4331$ & N317 & 005740.58 & 434732.30 & 5429 & 77.2 & 11.16 & 22.66 & 24.92 & 2.26 \\
\hline $00555+7614$ & & 005915 & 763052 & 4739 & 70.1 & 10.91 & 22.53 & 24.66 & 2.13 \\
\hline $01503+1227$ & & 015259.52 & 124227.90 & 4558 & 63.3 & 10.91 & 22.36 & 24.65 & 2.29 \\
\hline $01519+3640$ & & 015453.93 & 365504.40 & 5621 & 79.2 & 11.02 & 22.14 & 24.74 & 2.60 \\
\hline $01555+0250$ & ARP126 & 015805.26 & 030500.90 & 5431 & 75.6 & 10.94 & 22.47 & 24.68 & 2.20 \\
\hline $01556+2507$ & & 015830.63 & 252136.90 & 4916 & 68.8 & 10.99 & 22.36 & 24.75 & 2.39 \\
\hline $01579+5015$ & & 020109.65 & 503025.50 & 4875 & 69.5 & 10.85 & 22.29 & 24.55 & 2.26 \\
\hline $02071+3857$ & N828 & 021009.52 & 391125.30 & 5374 & 75.8 & 11.33 & 22.85 & 25.07 & 2.22 \\
\hline $02080+3725$ & N834 & 021101 & 373958 & 4593 & 64.7 & 10.95 & 22.49 & 24.69 & 2.20 \\
\hline $02208+4744$ & & 022408.00 & 475810.68 & 4679 & 66.5 & 11.09 & 22.51 & 24.84 & 2.33 \\
\hline
\end{tabular}

Notes. Columns: (1) IRAS name; (2) other name; (3) and (4) right ascension and declination (from VLA observations, unless undetected in which case IRAS positions are given in italics); (5) heliocentric velocity (cz) from NED; (6) luminosity distance (see Section 2); (7) FIR luminosity (see Section 3.3); (8) $1.4 \mathrm{GHz}$ luminosity calculated from the NRAO VLA Sky Survey (NVSS) catalog (Condon et al. 1998); (9) FIR spectral luminosity (see Section 3.3); (10) $q_{v}=\log \left(L_{\mathrm{FIR}} / L_{1.4}\right)$.

(This table is available in its entirety in a machine-readable form in the online journal. A portion is shown here for guidance regarding its form and content.)

Our observational results are presented in Section 4 and our discussion in Section 5. Finally, in Section 6 we list our major conclusions.

\section{THE COLA PROJECT AND PREVIOUS OBSERVATIONS}

The COLA project has the primary goal of determining the relationship between AGNs and other galactic properties (such as galactic structure, degree of interaction, and star formation activity) in a moderate luminosity FIR selected sample. The sample contains all the galaxies in the IRAS Point Source Catalog (Beichman et al. 1988) with (1) flux densities at $60 \mu \mathrm{m}$ greater than $4 \mathrm{Jy}$, (2) heliocentric velocities between 3500 and $7000 \mathrm{~km} \mathrm{~s}^{-1}$ (to minimize Malmquist bias), and (3) galactic latitude $|b|>10^{\circ}$ (to avoid confusion with galactic objects). The resulting sample has 217 galaxies of which 110 are located in the northern hemisphere (COLA-N) and 107 in the Southern hemisphere (COLA-S).

The COLA sample is selected at the same IR wavelength as the Revised Bright Galaxy Sample (RBGS; see Sanders et al. (2003)) but with a flux limit which is somewhat smaller (4 Jy versus $5.24 \mathrm{Jy}$ ) plus an additional redshift range selection. It follows that there is a significant overlap between the two samples with 65/110 (59\%) of the COLA-N sources also being members of the RBGS.

Table 1 provides a summary of the COLA-N sample (for a similar table describing the southern sample see Corbett et al. 2002). The luminosity distance $D_{L}$ given in Column 7 for each source was calculated in NASA/IPAC Extragalactic Database (NED) assuming the three attractor model of Mould et al. (2000) and the cosmological parameters derived from 5 year Wilkinson Microwave Anisotropy Probe (WMAP) data (Hinshaw et al. 2009), i.e., $\Omega_{m}=0.276, \Omega_{v}=0.726$, and $H_{o}=70.5 \mathrm{~km} \mathrm{~s}^{-1}$. Details on the calculation of infrared and radio luminosities presented in Table 1 are given in Section 3.3. Table 1 shows that most COLA sources have bolometric IR luminosities close to the boundary defining luminous infrared galaxies (at $L_{\mathrm{IR}}=10^{11} L_{\odot}$, Sanders \& Mirabel (1996)) with a median IR bolometric luminosity for COLA-N of $10^{11.01} L_{\odot}$. All but one source has a bolometric IR luminosity in the range $10^{10.5}<L_{\mathrm{IR}} / L_{\odot}<10^{11.7}$, the exception being the nearby ULIRG Arp 220.

Low resolution $1.4,2.5$, and $4.8 \mathrm{GHz}$ continuum observations of COLA-S sources have been made using the Australia Telescope Compact Array (ATCA). These radio observations were complemented with high resolution single baseline $2.3 \mathrm{GHz}$ snapshot observations obtained using the Australian Long Baseline Array (LBA). Optical spectroscopy for a large fraction of the southern sources was also obtained using the Dual Beam Spectrograph (DBS) on the $2.3 \mathrm{~m}$ telescope at the Mount Stromlo and Siding Springs Observatory. Detailed descriptions of the COLA-S radio and optical observations can be found in Corbett et al. $(2002,2003)$ respectively.

Corbett et al. (2002) detected 9 out of the 105 COLA-S galaxies in their LBA observations. Of these, eight showed radio emission stronger than that predicted by the IR-radio correlation. Moreover 7 of the 14 sources with a total radio flux density more than $1 \sigma$ above that predicted by the FIR-Radio correlation were LBA detections. Based on these results, Corbett et al. (2002) concluded that sources detected with the LBA exhibited a statistically significant radio excess relative to the non-detections. This excess persisted even after the subtraction of the radio emission from the detected core implying the existence of associated AGN-powered diffuse radio emission from structures larger than $\sim 15 \mathrm{pc}$.

The COLA-S optical data (Corbett et al. 2003) showed that in the southern sample the fraction of galaxies optically classified as Seyfert was $\sim 15 \%$. Of these only $55 \%$ were detected with the LBA suggesting the there may be two populations of Seyferts, one population with extended radio structures and compact radio cores and the other without. In this paper, we revisit these tentative conclusions in light of the larger number of longbaseline detected sources in COLA-N. 
Table 2

Summary of Radio Observations

\begin{tabular}{lccccc}
\hline \hline \multicolumn{1}{c}{ Date } & Array & $\begin{array}{c}\text { Frequency } \\
(\mathrm{GHz})\end{array}$ & $\begin{array}{c}\text { Beamsize } \\
(\mathrm{mas})\end{array}$ & Sources & $\begin{array}{c}\text { Typical rms } \\
\left.(\mu \mathrm{Jy} \mathrm{beam})^{-1}\right)^{\mathrm{d}}\end{array}$ \\
\hline 1998 Jun 23 & VLA BnA & 4.9 & $900 \times 400$ & $0^{\circ}<\delta \leqslant 30^{\circ}$ & 75 \\
2003 Jul 19 & VLA A & 4.9 & 400 & $\delta>30^{\circ}$ and CC & 75 \\
2005 Feb 27 & Eb-Wb-Arc & 4.9 & 1.8 & $10^{\circ}<\delta \leqslant 40^{\circ}$ & 25 \\
2005 May 2006 & Eb-Wb-Jb1 ${ }^{\mathrm{c}}$ & 4.9 & 18.1 & $0^{\circ}<\delta \leqslant 10^{\circ}$ and $\delta>40^{\circ}$ & 56 \\
\hline
\end{tabular}

Notes.

${ }^{a} \mathrm{CC}$ : sources known to be compact at $4.9 \mathrm{GHz}$ from the first VLA epoch.

${ }^{\mathrm{b}}$ For the VLA observations this is the typical FWHM of the restoring beam. For the VLBI observations this is the typical fringe spacing of the longest baseline.

${ }^{\mathrm{c}}$ Eb: Effelsberg, Wb: Westerbork, Ar: Arecibo, and Jb1: Jodrell Bank (Lovell). Baselines lengths are Eb-Wb=266 km, Eb-Jb1 = 699 km, and Eb-Ar $=$ $6901 \mathrm{~km}$.

${ }^{\mathrm{d}}$ rms noise has units of $\mu \mathrm{Jy}_{\text {beam }}{ }^{-1}$ for VLA observations but for VLBI observations refers to noise on most sensitive baseline in $\mu \mathrm{Jy}$.

\section{OBSERVATIONS}

\subsection{VLA Observations}

Snapshot radio continuum observations for the COLA-N sources were obtained using the VLA at $4.8 \mathrm{GHz}$ in two observing epochs each of $24 \mathrm{hr}$ in length (see Table 2). Sources with $0^{\circ}<\delta<30^{\circ}$ were observed on 1998 June 23 in the VLA BnA configuration, while sources with $\delta>30^{\circ}$ were observed on 2003 July 19 in the A configuration. In addition, to ensure that all compact sources were observed with the A-array, 12 sources found to be compact at $4.8 \mathrm{GHz}$ from the first epoch were reobserved in the second epoch. The data were reduced using AIPS in a standard manner. Radio maps of size $50^{\prime \prime} \times 50^{\prime \prime}$ centered at the IRAS positions were produced for all the sources using natural weighting. Typical FWHM beam-sizes at $4.8 \mathrm{GHz}$ were $\sim 0^{\prime \prime} 4$ and $0^{\prime \prime} 9 \times 0$ 0'. 4 for A and BnA configurations, respectively. The typical $\mathrm{rms}$ noise levels were $75 \mu \mathrm{Jy}$ beam $^{-1}$

\subsection{VLBI Observations}

VLBI $4.8 \mathrm{GHz}$ snapshot observations at data rate $1 \mathrm{Gbit} \mathrm{s}^{-1}$ per station were conducted for the COLA North sample using the most sensitive telescopes available in the European VLBI network (EVN). The first epoch on 2005 February 27/28 used $\mathrm{Eb}$ (Effelsberg), $\mathrm{Wb}$ (Westerbork), and $\mathrm{Ar}$ (Arecibo) for all sample sources within the declination range $10^{\circ}<\delta<40^{\circ}$ (defined by the Ar zenith-distance restrictions and minimum elevation at the European stations) with $\sim 15$ minutes of onsource time per target giving $\sigma=25 \mu \mathrm{Jy}$ on the $\mathrm{Ar}-\mathrm{Eb}$ baseline. The second epoch on 2005 May 6/7 used Eb, Wb, and Jb1 (Jodrell Bank, Lovell telescope) for all the sources with $0^{\circ}<\delta<10^{\circ}$ and $\delta>40^{\circ}$ with $\sim 15$ minutes of on-source time per target giving noise $\sigma=56 \mu \mathrm{Jy}$ on the Eb-Jb1 baseline. Gaps in the schedule were filled by re-observing 21 sources from the first epoch. In both epochs, strong nearby phase reference and amplitude calibrators ( $>500 \mathrm{mJy}$ ) were observed for 10 minutes every $\sim 1 \mathrm{hr}$. The Eb-Wb baseline ( $266 \mathrm{~km}$ ) which is common to both epochs has a fringe spacing of $46.5 \mathrm{mas}$ at $4.8 \mathrm{GHz}$, which is similar to the 47.4 mas fringe-spacing of the ATCA-Tidbinbilla baseline $(566 \mathrm{~km})$ at $2.3 \mathrm{GHz}$ used by Corbett et al. (2002) to observe the southern sample. Because of scheduling restrictions and bad data we only obtained useful VLBI observations for 90 sources.

AIPS was used for the initial data reduction and calibration stages with instrumental phase and delay being determined toward strong calibrators. Monitored system temperatures and gains were used to calibrate amplitudes. An adjustment of $\sim 30 \%$ was required on $\mathrm{Jb} 1$ to obtain consistent calibration on sources known to be unresolved from the VLBA calibrator database. Our final estimate is that quoted baseline flux densities are accurate to $\sim 15 \%$.

Fringe fitting was performed in AIPS on all the calibrators and then these solutions were applied to the interleaved target source scans. After this we exported the data to our own software within which Delay-Rate maps were made for each baseline/ target source. The size of these delay-rate maps were set such that a compact source would be detectable up to $20^{\prime \prime}$ from the correlation position. Finally, the Delay-Rate maps were transformed into R.A.-decl. offset maps relative to the phase center. These maps show only features which are smaller than the baseline fringe spacing (see Parra 2007). We adopted a detection threshold of 6 times the measured rms noise.

\subsection{Archival Data Radio and IR Data}

In addition to our own radio data our analysis made use of source $1.4 \mathrm{GHz}$ total flux densities taken from the NVSS catalog (Condon et al. 1998). Infrared total flux densities were taken from the IRAS Point Source Catalog (Beichman et al. 1988). In order to calculate source infrared to radio $(1.4 \mathrm{GHz})$ ratios $\left(q_{1.4}\right)$ as defined by Helou et al. (1985) we calculated for each source an equivalent FIR flux density from $S_{\mathrm{FIR}}=1.26 \times 10^{-14}\left(2.58 f_{60}+f_{100}\right) / 3.75 \times 10^{12} \mathrm{~W} \mathrm{~m}^{-2} \mathrm{~Hz}^{-1}$, where $f_{60}$ and $f_{100}$ are the cataloged 60 and $100 \mu \mathrm{m}$ IRAS flux densities. For comparison with COLA-S and other data sets for each source a FIR spectral luminosity (see Table 1, Column 9) was calculated assuming isotropic emission. Finally, bolometric IR $(8 \mu \mathrm{m}-1000 \mu \mathrm{m})$ luminosities in solar units were calculated (see Table 1, Column 7) from the IRAS flux densities using the relation given by Sanders \& Mirabel (1996).

\subsection{Optical Spectroscopy Observations}

Optical spectroscopy observations were taken using the 60 inch CfA telescope at Mt Hopkins, Arizona between 2001 February and October using the FAST spectrograph. A slit width of $3^{\prime \prime}$ was used, corresponding to $0.9 \mathrm{kpc}$ at the median sample source distance. A grating of 300 lines $/ \mathrm{mm}$ was used to disperse light between 4000 and $7000 \AA$ and gave a spectral resolution of $6 \AA$. A single exposure of between 900 and $1200 \mathrm{~s}$ was made for each target. Spectrophotometric and smooth standards (often the same stars) were observed during each night and used to 

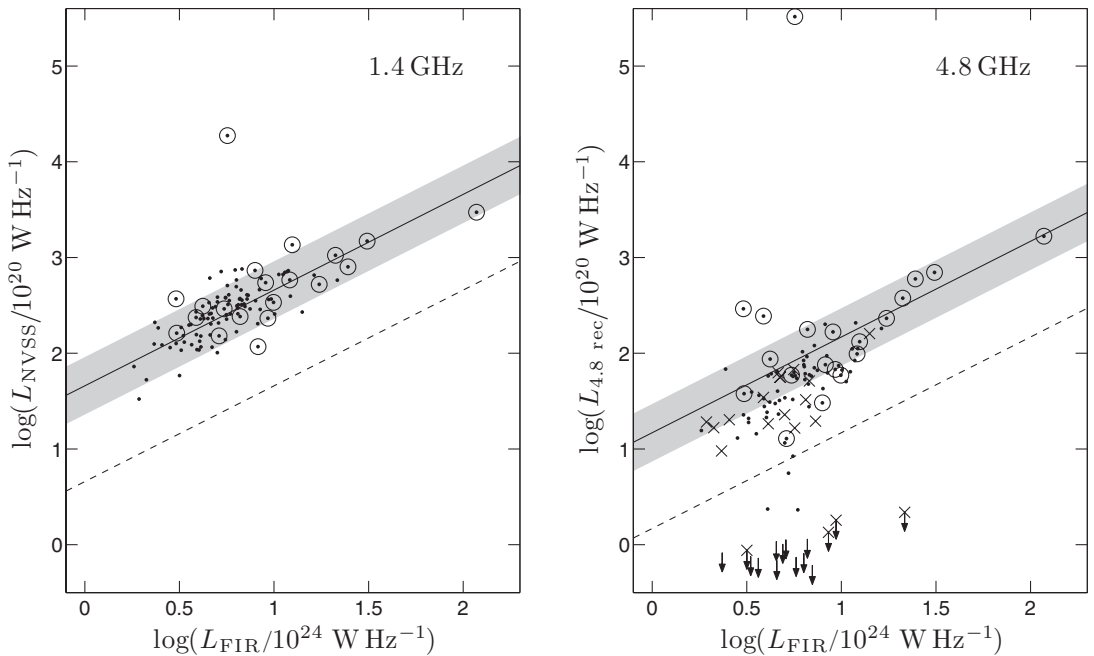

Figure 1. FIR to radio correlation plots for the COLA North sample at $1.4 \mathrm{GHz}$ and at $4.8 \mathrm{GHz}$. In both panels sources detected by VLBI are shown circled. Left: $1.4 \mathrm{GHz}$ radio spectral luminosity calculated using NVSS fluxes vs. FIR spectral luminosity $L_{\mathrm{FIR}}$ (see Section 3.3). Most of the COLA North sources are tightly clustered around the median $q_{1.4}=2.34$ estimated for a sample of 1809 galaxies by Yun et al. (2001) which is shown by a solid line. Note on average that for the sample $L_{\mathrm{FIR}}=1 \times 10^{25} \mathrm{WHz}^{-1}$ corresponds to $L_{\mathrm{IR}}=10^{11.25} L_{\odot}$. Right: $4.8 \mathrm{GHz}$ spectral luminosity calculated using the recovered $4.8 \mathrm{GHz}$ fluxes from our VLA maps (see Section 3.1) vs. $L_{\mathrm{FIR}}$. Arrows indicate $5 \sigma$ upper limits for the 15 sources not detected in these observations. Crosses indicate the 20 sources having no VLBI data. The median $q_{4.8}=2.83$ measured by Wunderlich et al. (1987) from a sample of 99 normal spirals is shown by a solid line. In both panels, the gray area defines the region of \pm 0.3 dex around the median and a dashed line is drawn at -1 dex.

flux-calibrate and correct for atmospheric absorption features in the spectra. We were able to measure useful line ratios for $61 \%$ of the sample.

Data reduction was carried out in the standard manner using IRAF. The frames were de-biased and flat fielded. Galaxy spectra were extracted using an aperture centered on the position of peak flux across the slit. If more than one peak was seen in the spatial direction a spectrum was extracted at the position of each peak. Sky-subtraction was then carried out and wavelength calibration done using an $\mathrm{Ne}-\mathrm{Ar}$ arc lamp. The observations were then corrected for atmospheric extinction and flux calibrated using the spectrum of a spectrophotometric standard. Finally, atmospheric absorption features in the target spectra were removed using the smooth spectrum standard observed at a similar air mass to the target.

The line fluxes were measured by fitting (multiple) Gaussians to the emission lines using NGAUSSFIT in IRAF. The continuum flux was subtracted using a linear fit to small portions of the continuum either side of the emission line. The $\mathrm{H} \alpha(\lambda 6562 \AA)$ and [N II] doublet ( $\lambda \lambda{ }^{6} 6583,6548 \AA$ ) were often overlapping, in these cases line profiles were de-blended by simultaneously fitting a separate Gaussian for each line. When $\mathrm{H} \alpha$ was present in absorption as well as emission, both the emission and absorption features were fitted simultaneously with Gaussians.

\section{RESULTS}

\subsection{Sample Overall Radio/FIR Ratios}

In a FIR-selected sample of galaxies such as COLA, it is expected that in most sources star formation will be the dominant mechanism powering the FIR emission. For such sources radio and FIR luminosities and flux densities are expected to be tightly correlated (see Condon 1992). The total $1.4 \mathrm{GHz}$ and FIR luminosities for our sample are plotted in Figure 1 (left). Except for one outlier (3C84) which is unusually radio luminous, sources tightly follow the expected correlation. We calculated for each source the logarithmic ratio of flux densities $q_{1.4}=\log \left(S_{\mathrm{FIR}} / S_{1.4}\right)$ as defined by Helou et al. (1985). Excluding $3 \mathrm{C} 84$, the mean value of $q_{1.4}$ is $2.34 \pm 0.01$ (and the median $2.31 \pm 0.01$ ) which is in good agreement with the mean $q_{1.4}=2.34 \pm 0.01$ measured by Yun et al. (2001) from a sample of 1809 galaxies (solid line in Figure 1). In contrast the median value for COLA-S (Corbett et al. 2002) is $q_{1.4}=2.43 \pm 0.03$ which is significantly $(4 \sigma)$ larger than the COLA-N median value.

To try to resolve the above discrepancy we compared NVSS and COLA fluxes for the 56 COLA South sources also contained in the NVSS catalog (i.e., those with $\delta \geqslant-40^{\circ}$ ). We found NVSS flux densities for these sources which were $12 \%$ larger than those measured by Corbett et al. (2002), resulting in $q_{1.4}=2.36 \pm 0.05$ for ATCA fluxes and $2.31 \pm 0.04$ for NVSS fluxes. It has been noted by Prandoni et al. (2000), Norris et al. (2006), and Kellermann et al. (2008) that for sources with flux densities $\lesssim 15 \mathrm{mJy}$ NVSS flux densities tend to be $\sim 10 \%$ larger than ATCA measurements. Although COLA total flux densities are about a factor of two above this flux limit the effect is neither understood nor well defined and so this scale difference may contribute to the apparent difference of median $q_{1.4}$. We conclude that the difference in $q_{1.4}$ between COLA-N and COLA-S is likely caused by (1) a $\sim 10 \%$ calibration difference for weak sources between NVSS and ATCA flux densities, combined with (2) a small number of sources at $\delta<-40^{\circ}$ which have anomalously large values of $q_{1.4}$, perhaps simply because of small number statistics.

It remains unclear whether the likely $\sim 10 \%$ calibration difference between Northern and Southern hemispheres noted above for weak sources is a property of NVSS specifically or all VLA observations. Furthermore, the $4.8 \mathrm{GHz}$ measurements of galaxies made with the Bonn $100 \mathrm{~m}$ single dish give $q_{4.8}=2.71 \pm 0.03$ (Wunderlich et al. 1987), assuming a spectral index of 0.75 this then gives an estimated $q_{1.4}=2.31 \pm 0.03$ close to the VLA value, supporting the idea that Northern hemisphere $q_{1.4}$ values are consistent with each other. Given the importance of sorting out the origin of radio flux scale differences between hemispheres it seems that dedicated simulta- 

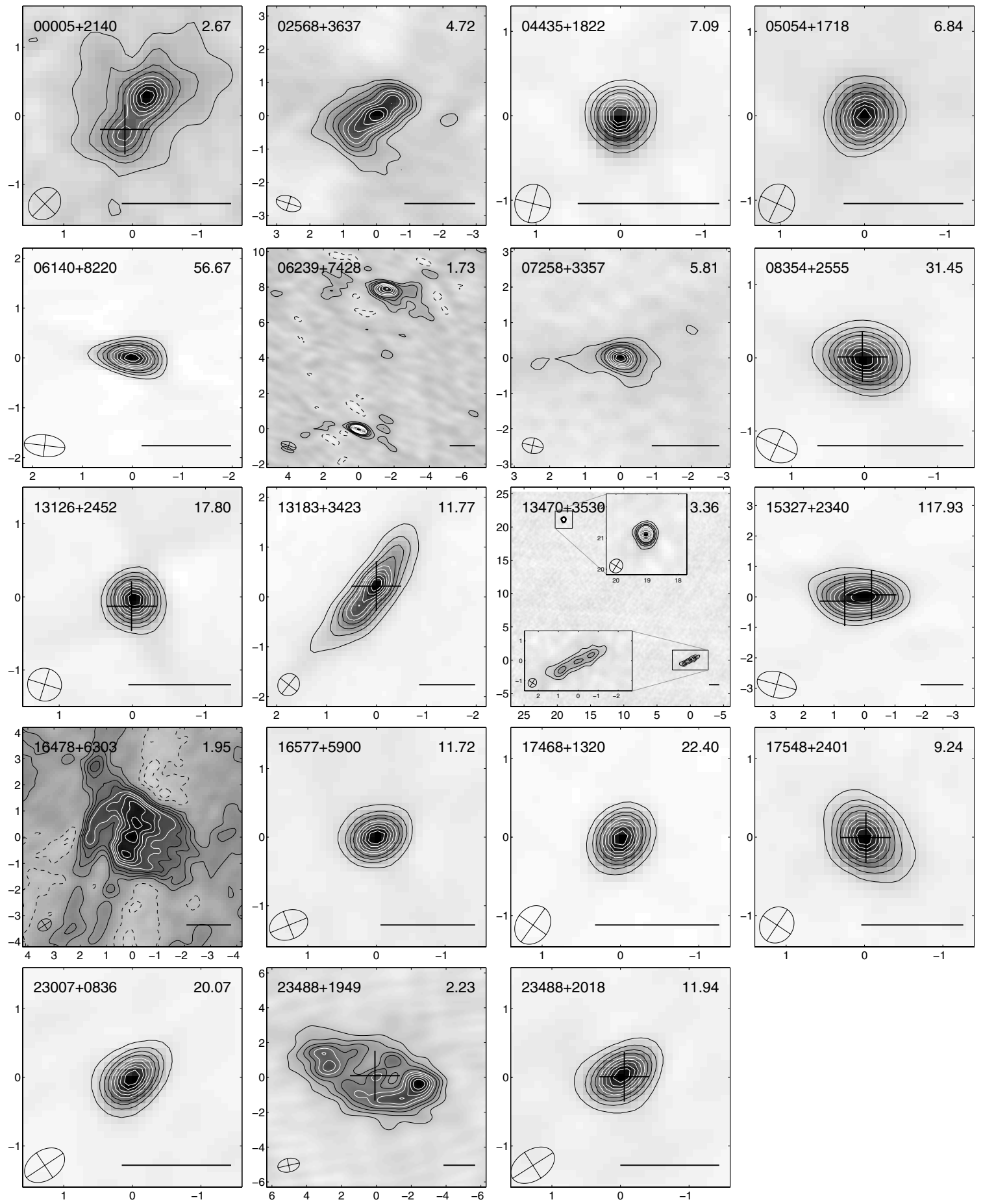

Figure 2. VLA $4.8 \mathrm{GHz}$ naturally weighted maps for 19 of the 20 VLBI detected sources (the well known core-dominated source $031643+4119$ (3C 84 ) is omitted). VLA images of the sources undetected by VLBI are given in Figure 3. Shown are the highest resolution images of each source which in all cases come from A-array observations except for 15327+2340 (Arp220) and 23488+1949 which come from BnA observations. In 05336+5407 two features are detected 30" apart, only the brighter more compact NW feature which contains the VLBI source is shown. In all of the figures, the IRAS source name is indicated in the top left-corner. Peak brightness in $\mathrm{mJy} \mathrm{beam}^{-1}$ is indicated in the top right corner. Black contours are drawn at $10 \%, 20 \%, 30 \%$, and $40 \%$ of the peak brightness. White contours are drawn at $50 \%, 60 \%, 70 \%, 80 \%$, and $90 \%$ of the peak brightness. Negative contours are drawn with dashed lines at $-10 \%,-20 \%,-30 \%$, and $-40 \%$ of the peak brightness. A cross indicates the position of the VLBI core detections for those source having observations using the Eb-Ar baseline. Axes tick-marks are in arcseconds. The horizontal line on the bottom-right corner indicates $500 \mathrm{pc}$ at the distance of the source.

neous ATCA/VLA observations of sources with different flux densities should be made.

\subsection{VLA 4.8 GHz Maps}

The results of our VLA observations are summarized in Table 3. We successfully detected and mapped 95 out of the
110 observed sources. The 15 non-detections are probably large galaxies with a surface brightness too low to be detected with the VLA configuration used.

The images for the VLA detections are shown in Figures 2 and 3 with the former showing sources which are also VLBI detections and the latter showing those that are VLBI non-detections. 

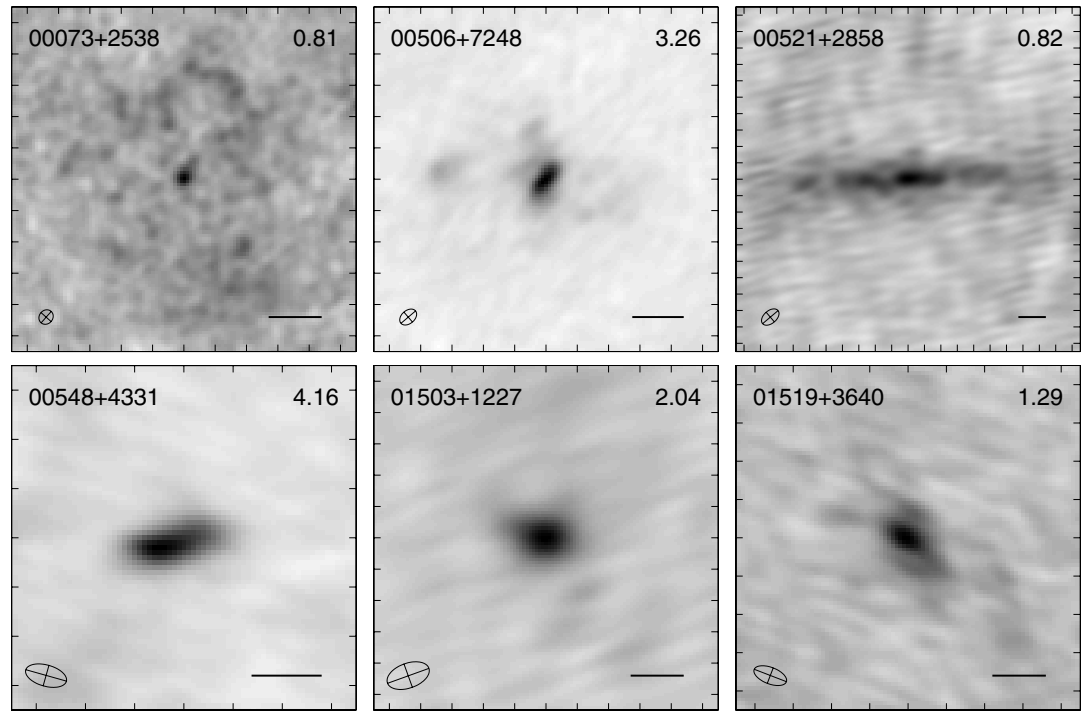

Figure 3. VLA 4.8 GHz naturally weighted maps for the VLA detected sources of the COLA North sample without VLBI detections. Maps for the VLBI detections are given in Figure 2. Column 2 in Table 3 gives the VLA array used, for sources observed in two arrays the higher resolution image is shown. IRAS names are indicated on the top-left corner of every panel. Peak brightness in mJy beam ${ }^{-1}$ is indicated on the top-right corner. Tick marks are separated by 1 arcsec with the restoring beam shown at the bottom-left corner. The horizontal line at the bottom-right corner is $500 \mathrm{pc}$ at the distance of the source. Only the first six images are shown here and the remaining images can be found in the online version.

(An extended version of this figure is available in the online journal.)

The images show a wide variety of morphologies. Following Neff \& Hutchings (1992) we have classified the sources into four different morphological classes: Core (3), Core and Extensions (CE), Extended with no core (E), and Undetected (U), each class containing $28,62,5$, and 15 sources, respectively. There are two sources with clear ring structures, namely IRAS05091+0508 (NGC 1819) and 23488+1949 (NGC 7771); there are an additional four showing elongated linear structures typical of edgeon disks.

Total recovered fluxes at $4.8 \mathrm{GHz}\left(S_{4.8 t o t a l}\right)$ were obtained by integrating the flux over a region enclosed by the lowest reliable contour of radio emission. The median $q_{4.8}=\log \left(L_{\mathrm{FIR}} / L_{4.8 \mathrm{tot}}\right)$ obtained for the sample is $3.03 \pm 0.05$ which is significantly higher than the $2.71 \pm 0.03$ measured by Wunderlich et al. (1987) using single dish observations of a sample of 99 normal spirals. We conclude that we are on average resolving out $\sim 50 \%$ of the $4.8 \mathrm{GHz}$ flux density. We note however that for those sources with compact central features the fraction of missing flux density is $\lesssim 20 \%$.

\subsection{VLBI Detections}

We detect, on at least one VLBI baseline, 20 out of 90 sources observed. The measured flux densities on each baseline are given in Table 4. On the shortest Eb-Wb baseline (see Figure 4) the signal-to-noise ratio for most detections is generally significantly larger than the $6 \sigma$ detection threshold (see Figure 4 ) while non-detections are bounded to $<4 \sigma$. It is notable that we obtain VLBI detections for five of the six sources with IR luminosities above $10^{11.4} L_{\odot}$. A histogram showing the VLBI detections and non-detections versus IR luminosity is presented in Figure 5 (top) which shows that the probability of detecting a VLBI core increases with IR luminosity.

Table 4 shows that there are nine sources with simultaneous measurements on the $\mathrm{Eb}-\mathrm{Wb}$ and $\mathrm{Eb}-\mathrm{Jb} 1$ baselines. Of these, seven have similar fluxes (within the noise) on these two baselines, the latter of which is $\sim 2.6$ times longer than the first, showing that the VLBI component is unresolved.
Of the 14 sources with simultaneous $\mathrm{Eb}-\mathrm{Wb}$ and $\mathrm{Eb}-\mathrm{Ar}$ observations, 9 are detected on the $\mathrm{Eb}-\mathrm{Ar}$ baseline, but with significantly reduced flux density, indicating that the VLBI sources are resolved on the longest baseline. We estimate an angular size (see Table 4) by approximating the flux ratio between both baselines to that expected from a circular Gaussian (see Pearson 1999). The high sensitivity and small fringe spacing of the Eb-Ar baseline resulted in a very small uncertainty on the phase center offsets of the detections. These VLBI position offsets are shown as crosses on the corresponding VLA map in Figure 2.

\subsection{FIR/Radio Ratios of the VLBI Detections}

Figure 1 (left) shows that the total $1.4 \mathrm{GHz}$ VLA flux densities of our VLBI detected sources follow the FIR to radio correlation. There are $15 / 20$ detections within \pm 0.3 dex of the empirical line defined by Yun et al. (2001) (i.e., within the gray region shown). Of the remainder two are just slightly above and two slightly below the \pm 0.3 dex line. Only one source, $3 \mathrm{C} 84$, is significantly above the correlation. At $4.8 \mathrm{GHz}$ a similar trend is observed with $14 / 20$ detections located within \pm 0.3 dex of the empirical line defined by Wunderlich et al. (1987). Consistent with the above results we find the median $q_{1.4}$ for the VLBI detections and non-detections to be indistinguishable (i.e., $2.30 \pm 0.08$ and $2.31 \pm 0.02$ respectively). Likewise, if we omit 3C84 the mean of the VLBI detection is $2.33 \pm 0.08$ and of the non-detections is $2.32 \pm 0.02$. We can conclude that the data show that excepting 3C84 the total radio flux density is dominated by star formation regardless of whether or not a VLBI core is present.

The above results appear to differ from those in COLA-S where a difference in median $q_{1.4}$ between VLBI detections and non-detections was claimed. From Table 3 of Corbett et al. (2002) the median $q_{1.4}$ value and its estimated one sigma error for the nine definite COLA-S VLBI detections was $2.07 \pm 0.17$ while for the VLBI non-detections it was $2.44 \pm 0.03$. The errors on theses estimates are calculated from the internal dispersion about the median $q_{1.4}$ for the two sub-samples as given in Corbett 
Table 3

Radio and Optical Observations and Results

\begin{tabular}{|c|c|c|c|c|c|c|c|c|c|}
\hline $\begin{array}{c}\text { IRAS } \\
\text { Name } \\
(1)\end{array}$ & $\begin{array}{c}\text { VLA } \\
\text { Array } \\
(2)\end{array}$ & $\begin{array}{c}\text { VLA } \\
\text { Morph } \\
\text { (3) }\end{array}$ & $\begin{array}{c}S_{4.8 t o t a l} \\
(\mathrm{mJy}) \\
(4)\end{array}$ & $\begin{array}{l}q_{4.8} \\
(5)\end{array}$ & $\begin{array}{c}S_{4.8 \text { comp }} \\
\text { (mJy) } \\
(6)\end{array}$ & $\begin{array}{c}\theta_{M} \times \theta_{m} \\
\left({ }^{\prime \prime} \times{ }^{\prime \prime}\right) \\
(7)\end{array}$ & $\begin{array}{c}\log T_{b} \\
(\mathrm{~K}) \\
(8)\end{array}$ & $\begin{array}{c}\text { VLBI } \\
\text { Epoch } \\
(9)\end{array}$ & $\begin{array}{c}\text { Optical } \\
\text { Class } \\
(10)\end{array}$ \\
\hline $00005+2140$ & $\mathrm{BnA}-\mathrm{A}$ & $\mathrm{CE}$ & 5.66 & 2.97 & 0.00 & $\ldots$ & $\ldots$ & $1 *$ & S \\
\hline $00073+2538$ & $\mathrm{BnA} \ldots \mathrm{A}$ & $\mathrm{C}$ & 18.40 & 2.85 & 1.23 & $0.45 \times 0.22$ & 2.63 & 1 & $\mathrm{C}$ \\
\hline $00506+7248$ & A & $\mathrm{CE}$ & 31.60 & 2.95 & 13.60 & $1.27 \times 0.66$ & 2.75 & 2 & $\ldots$ \\
\hline $00521+2858$ & $\mathrm{BnA}$ & $\mathrm{E}$ & 6.07 & 3.13 & 3.40 & $3.15 \times 0.91$ & 1.61 & 1 & $\ldots$ \\
\hline $00548+4331$ & A & $\mathrm{CE}$ & 13.21 & 2.95 & 13.11 & $1.51 \times 0.41$ & 2.87 & 2 & $\ldots$ \\
\hline $00555+7614$ & A & $\mathrm{U}$ & $\ldots$ & 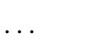 & .. & $\ldots$ & $\ldots$ & 2 & $\ldots$ \\
\hline $01503+1227$ & $\mathrm{BnA}$ & $\mathrm{CE}$ & 6.23 & 3.18 & 5.25 & $1.58 \times 1.03$ & 2.05 & 1 & $\mathrm{C}$ \\
\hline $01519+3640$ & A & $\mathrm{CE}$ & 4.38 & 3.23 & 3.92 & $1.13 \times 0.74$ & 2.21 & $1-2$ & $\mathrm{C}$ \\
\hline $01555+0250$ & $\mathrm{BnA}$ & $\mathrm{CE}$ & 8.23 & 2.92 & 1.85 & $2.04 \times 1.06$ & 1.47 & $\ldots$ & $\mathrm{L}$ \\
\hline $01556+2507$ & $\mathrm{BnA}$ & $\mathrm{CE}$ & 11.16 & 2.95 & 7.37 & $1.32 \times 1.02$ & 2.28 & 1 & $\mathrm{H}$ \\
\hline $01579+5015$ & A & $\mathrm{CE}$ & 2.49 & 3.39 & 1.82 & $0.59 \times 0.38$ & 2.45 & 2 & $\mathrm{~L}$ \\
\hline $02071+3857$ & $\mathrm{~A}$ & E & 12.20 & 3.15 & 0.93 & $0.85 \times 0.56$ & 1.83 & 2 & $\ldots$ \\
\hline $02080+3725$ & A & $\mathrm{U}$ & $\ldots$ & $\ldots$ & .. & $\ldots$ & $\ldots$ & $1-2$ & $\mathrm{H}$ \\
\hline $02152+1418$ & $\mathrm{BnA}$ & $\mathrm{CE}$ & 2.39 & 3.82 & 1.34 & $1.49 \times 0.94$ & 1.52 & $1-2$ & $\ldots$ \\
\hline $02208+4744$ & A & $\mathrm{CE}$ & 10.92 & 3.08 & 10.61 & $1.78 \times 1.02$ & 2.31 & 2 & $\ldots$ \\
\hline $02253+1922$ & $\mathrm{BnA}$ & $\mathrm{U}$ & $\ldots$ & $\ldots$ & $\ldots$ & $\ldots$ & $\ldots$ & $1-2$ & $\mathrm{H}$ \\
\hline
\end{tabular}

Notes. Columns: (1) IRAS Name; (2) VLA array(s) used; (3) morphology class of VLA 4.8 GHz image. C-Compact, E-Extended, CE-Compact plus Extended, CER-Compact plus extended with ring, U-Undetected; (4) recovered $4.8 \mathrm{GHz}$ flux density in VLA image; (5) log of ratio of $4.8 \mathrm{GHz}$ recovered VLA flux density to FIR flux density; (6) total flux density of Gaussian fitted to brightest feature in VLA image (after removing VLBI component, hence zero indicates that brightest feature was dominated by the VLBI component); (7) fitted Gaussian major and minor axes to peak VLA feature (FWHM arcseconds), note for IRAS15327+2340 (Arp 220) these are for the western nucleus as taken from the literature (see Section 4.5); (7) fitted Gaussian major and minor axes to peak VLA feature (FWHM arcseconds); (8) peak $4.8 \mathrm{GHz}$ brightness temperature of Gaussian component (see Section 4.5), note if formally unresolved along one axis a 50 mas FWHM was assumed along that axis); (9) VLBI Epoch(s) observed (asterisk indicates VLBI detection, see Table 4 for details); (10) optical class (see Section 4.6) with $\mathrm{H}=\mathrm{HII}, \mathrm{S}=$ Seyfert, $\mathrm{L}=\mathrm{LINER}$, and C $=$ Composite.

(This table is available in its entirety in a machine-readable form in the online journal. A portion is shown here for guidance regarding its form and content.)

et al. (2002). We ascribe this apparent two sigma difference between the two sub-samples in the South as being due to the lower VLBI sensitivity in the South combined with the overall form of the $q_{1.4}$ distribution. This distribution has a tail of radio core-dominated, low $q_{1.4}$ sources (such as 3C84 in the North and NGC5793 in the South) which are easily VLBI detected plus weaker VLBI cores in galaxies whose radio emission is star formation dominated. The lower sensitivity VLBI observations in the South contain a larger fraction of the former class of source which reduces the estimated $q_{1.4}$ of VLBI detection compared to non-detections. Specifically, in the South four out of nine VLBI detected sources have $q_{1.4} \lesssim 2$ while the fraction is only 3 out of 20 in the North. Because low $q_{1.4}$ sources comprise almost half of the VLBI detected sample in the South they can bias the median $q_{1.4}$ while in contrast in the North such outlier sources have negligible effect.

\subsection{Relation Between VLA and VLBI Sources}

Visual inspection of the VLA radio maps indicates a bias for VLBI detections to be in sources with compact structure and high peak brightness (see Figure 2). This is confirmed in terms of VLBI detection rate versus morphology class (see Section 4.2). Among the VLBI observed sources there are 13/27 detections of class C, $6 / 48$ of class $C E$, and $1 / 5$ of class $E$. We do not detect any class $\mathrm{U}$ source. This result was confirmed by plotting VLA visibility amplitude versus $u v$ distance. The majority of VLBI detections were indeed found in sources with compact, but not unresolved, arcsecond-scale structures. The size of these structures was estimated by subtracting an unresolved source equivalent to the VLBI detection from the brightest component in each VLA map and then fitting a Gaussian to the difference.

In two cases $(00005+2140$ and $06239+7428)$, the VLBI core subtraction removed all the flux density implying that the brightest VLA component was caused completely by the VLBI source itself. We consider these two sources together with $03164+4119$ (3C84) to be VLBI core-dominated sources and omit them from the subsequent analysis. For the rest of the sources the task JMFIT was used to fit a Gaussian within a tight box containing the remaining feature. For the vast majority of sources in classes $\mathrm{C}$ and $\mathrm{CE}$ with a clear compact component there was no ambiguity in the Gaussian fitting, i.e.,, the results of the fits were the same regardless of the size of the fitting box. For sources in class $\mathrm{E}$, the size estimates were found to vary by up to a factor of $\sim 2$ depending on the chosen box. However, in all such cases the fitted sizes were large $\left(\gtrsim 2^{\prime \prime}\right)$. The resulting component flux densities and major and minor axes $\theta_{M}$ and $\theta_{m}$ are listed in Columns 6 and 7 of Table 3.

Peak brightness temperatures $T_{b}$ at $4.8 \mathrm{GHz}$ for the compact VLA structures were calculated using the relation $T_{b} \simeq 35 \times$ $\left(S_{4.8 \text { peak }}\right)\left(\theta_{M} \times \theta_{m}\right)^{-1} \mathrm{~K}^{\circ}$ (Column 8 in Table 3$)$. In a few sources the fitted deconvolved minor axis was zero; in these cases its size was set to 50 mas which was equal to the smallest size found in other sources.

Uniquely, among sources with compact structure we did not collect any VLA A-array data for the well known ULIRG source $15327+2340$ (Arp220). In order to treat this source in a similar way to the rest we give in Table 3 radio component properties from the literature for the brighter western nucleus (sizes from Condon et al. 1991 with flux densities scaled to $5 \mathrm{GHz}$ using the spectral index of Rovilos et al. 2003). 
Table 4

VLBI Results

\begin{tabular}{|c|c|c|c|c|c|c|c|}
\hline $\begin{array}{l}\text { COLA } \\
\text { Name } \\
(1) \\
\end{array}$ & $\begin{array}{l}\text { Other } \\
\text { Name } \\
(2)\end{array}$ & $\begin{array}{c}\begin{array}{c}\mathrm{Eb}-\mathrm{Wb} \\
(\mathrm{mJy}) \\
(3)\end{array} \\
\end{array}$ & $\begin{array}{c}\mathrm{Eb}-\mathrm{Jb} 1 \\
(\mathrm{mJy}) \\
(4)\end{array}$ & $\begin{array}{c}\mathrm{Eb}-\mathrm{Ar} \\
(\mathrm{mJy}) \\
(5)\end{array}$ & $\begin{array}{l}\text { Angular Size } \\
\text { (mas) } \\
(6)\end{array}$ & $\begin{array}{c}\text { Linear Size } \\
(\mathrm{pc}) \\
(7) \\
\end{array}$ & $\begin{array}{c}\text { Optical } \\
\text { Class } \\
(8) \\
\end{array}$ \\
\hline $00005+2140$ & Mrk 334 & $1.67(0.15)$ & & $1.02(0.03)$ & 0.68 & 0.29 & $\mathrm{~S}$ \\
\hline $02568+3637$ & & $1.61(0.14)$ & $1.92(0.16)$ & & & & $\mathrm{S}$ \\
\hline $03164+4119$ & $3 \mathrm{C} 84$ & & & $15500(0.02)$ & & & $\mathrm{L}$ \\
\hline $04435+1822$ & & $3.32(0.14)$ & $\ldots$ & $3.10(0.03)$ & 0.25 & 0.07 & $\mathrm{H}$ \\
\hline $05054+1718$ & & $1.64(0.18)$ & $\ldots$ & $<0.20$ & $>1.41$ & $>0.50$ & S \\
\hline $06140+8220$ & & $4.12(0.32)$ & $\ldots$ & $\ldots$ & & & $\ldots$ \\
\hline $06239+7428$ & & $\ldots$ & $1.51(0.19)$ & $\begin{array}{l}\cdots \\
\cdots\end{array}$ & & & $\begin{array}{l}\cdots \\
\cdots\end{array}$ \\
\hline F07258+3357 & N2388 & $1.38(0.18)$ & $1.26(0.14)$ & $<0.20$ & $>1.35$ & $>0.35$ & $\mathrm{H}$ \\
\hline $08354+2555$ & Arp 243 & $2.03(0.10)$ & $\ldots$ & $0.27(0.02)$ & 1.38 & 0.49 & $\ldots$ \\
\hline $13126+2452$ & & $1.38(0.17)$ & $\ldots$ & $0.88(0.04)$ & 0.65 & 0.16 & - \\
\hline $13183+3423$ & Arp 193 & $2.17(0.17)$ & $3.85(0.15)$ & $0.70(0.04)$ & 1.03 & 0.46 & C \\
\hline $13470+3530$ & & $1.79(0.11)$ & $5.64(0.16)$ & $<0.18$ & $>1.47$ & $>0.48$ & $\ldots$ \\
\hline $15327+2340$ & Arp 220 & $4.37(0.17)$ & $\ldots$ & $\begin{array}{l}2.68(0.04) \\
1.02(0.04)\end{array}$ & 0.40 & 0.14 & $\mathrm{~L}$ \\
\hline $16478+6303$ & N6247 & $2.60(0.12)$ & $<0.82$ & $\ldots$ & & & $\ldots$ \\
\hline $16577+5900$ & Arp 293 & $6.11(0.27)$ & $5.36(0.17)$ & $\ldots$ & & & $\mathrm{C}$ \\
\hline $17468+1320$ & & $8.33(0.21)$ & $5.31(0.13)$ & $<0.25$ & $>1.82$ & $>0.57$ & $\ldots$ \\
\hline $17548+2401$ & & $4.45(0.20)$ & $\ldots$ & $0.39(0.04)$ & 1.52 & 0.58 & L \\
\hline $23007+0836$ & N7469 & $5.12(0.22)$ & $6.32(0.15)$ & $\ldots$ & & & S \\
\hline $23488+1949$ & N7771 & $<1.14$ & $\ldots$ & $0.33(0.04)$ & $<1.08$ & $<0.28$ & $\mathrm{~L}$ \\
\hline $23488+2018$ & Mrk 331 & $5.02(0.21)$ & $4.92(0.13)$ & $0.30(0.04)$ & 1.63 & 0.57 & C \\
\hline
\end{tabular}

Notes. Columns: (1) IRAS Name (from Point Source Calalog, excepting F07258+3357 which is taken from the Faint Source Catalog); (2) other name; (3)-(5) VLBI flux and $1 \sigma$ uncertainty (in brackets) obtained from the peak in the Delay-Rate map. Upper limits for the non-detections are indicated at $5 \sigma$. A dash is shown for sources not observed at that baseline. For IRAS15327+2340 (Arp 220) on the longer baselines the two rows give results for the west and east nuclei, respectively; (6) FWHM of a circular Gaussian source fitting the flux ratio between the Eb-Wb and Eb-Ar baselines (see Section 4.3). Sources with flux upper limits on the Eb-Ar have consequently size lower limits; (7) corresponding fitted Gaussian FWHM linear sizes; (8) optical class (see Section 4.6) with $\mathrm{H}=\mathrm{HII}, \mathrm{S}=$ Seyfert, $\mathrm{L}=$ LINER, and C = Composite; a dash indicates no optical line data.

As can be seen from Figure 5 (bottom panel) VLBI detections seem to be strongly correlated with those sources whose VLA fitted components (after removing any VLBI contribution) have the highest $T_{b}$. VLBI detectability is less strongly correlated with large $L_{\mathrm{IR}}$ (top panel). Two-sided Kolmogorov-Smirnov tests comparing the distribution of VLBI detected and non-detected sources in $L_{\mathrm{IR}}$ and $T_{b}$ in each case rejects the hypothesis that VLBI/non-VLBI sources are drawn from the same population at probability levels of $P=0.82$ and $P=0.98$, respectively. This result demonstrates that the stronger correlation for VLBI detectability is with $T_{b}$ rather than with $L_{\mathrm{IR}}$.

\subsection{Optical Spectroscopy Classifications}

To classify sources based on their line ratios from our optical spectroscopy observations (see Section 3.4) we used the method of Kewley et al. (2006). This scheme first classifies sources into H II galaxies, AGN/star formation composites and pure AGN based on a mixture of theoretical (maximal starburst, Kewley et al. 2001) and empirical line-ratio boundaries (Kauffmann et al. 2003) delineating pure star-forming galaxies. These boundaries are shown respectively as solid and dashed curves delimiting the bottom left corners of the diagnostic line ratio diagrams in Figure 6. H II galaxies (class $\mathrm{H}$ ) are to the left and below all the curves including the dashed line in Figure 6(a). Composite sources (class C) lie between the solid and dashed lines in the $[\mathrm{N} \mathrm{II}] / \mathrm{H} \alpha$ diagram and AGN are above and to the right of the curves in all diagrams. The AGN population is further divided between Seyfert (class S) or LINER (class L) using the $[\mathrm{SII}] / \mathrm{H} \alpha$ and $[\mathrm{OI}] / \mathrm{H} \alpha$ diagnostic diagrams and the empirical results of Kewley et al. (2006) who found two well defined populations tracing different position angles on these diagrams. For a significant number of our sources either the [SII] or [O I] lines were not detected. In order to increase the number of detections we therefore used the same 2-of-3 criterion for classification as Yuan et al. (2010) whereby if there was no data for a given line ratio or if one diagram gave a classification inconsistent with the other two we chose the classification given by the majority of the diagrams.

Our available optical data allowed for the classification of 66 sources as summarized in Column 10 of Table 3. The fraction of different classifications agrees well with that found among sources of similar luminosity by Yuan et al. (2010). For the subset of sources observed by VLBI the compact radio core detection rates for each optical category are; Seyferts 4/8 (50\%), LINERS 4/6 (66 \%), Composite 3/20 (15\%), and $\mathrm{H}$ II 2/15 (14\%). These VLBI detection fractions are broadly consistent with the idea that VLBI cores trace AGN activity since the detection fraction is largest among AGN optical types (see Section 5.4).

\section{DISCUSSION}

\subsection{Nature of Detected VLBI Sources}

The primary objective of our VLBI observations was to detect compact cores indicating AGN activity. Here we discuss whether our 20 VLBI detections can be taken as unambiguous signs of AGN activity or whether they might be confused by the high spatial frequency tail of arcsecond scale diffuse emission, radio $\mathrm{SNe}$, or supernova remnants (SNRs). 


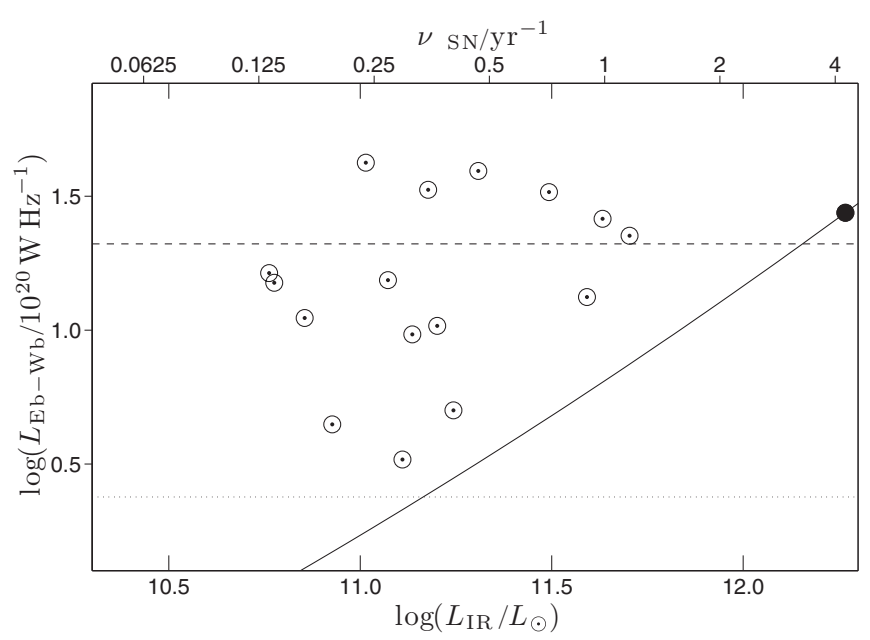

Figure 4. Radio spectral luminosity at $4.8 \mathrm{GHz}$ on the $\mathrm{Eb}-\mathrm{Wb}$ baseline for VLBI detected sources (excluding 3C84) vs. IR luminosity. The source Arp 220 is shown as a filled circle. The scale on the upper horizontal axis indicates the core-collapse supernova $(\mathrm{SN})$ rate $\nu_{\mathrm{SN}}$ estimated from the IR luminosity using the formula in van Buren \& Greenhouse (1994) (after applying a small FIR to IR luminosity correction). The luminosity at maximum light measured for the powerful Type IIn SN1986J is indicated with a horizontal dashed line at $2.1 \times 10^{21} \mathrm{~W} \mathrm{~Hz}^{-1}$. The diagonal line represents the spectral luminosity detected on the Eb-Wb baseline for Arp 220 scaled by IR luminosity. The lower horizontal dotted line indicates our typical $6 \sigma=780 \mu \mathrm{Jy}$ detection threshold on this baseline at the median distance of the COLA sample.

\subsubsection{VLBI Brightness Temperature and Diffuse Starburst Emission}

Could the VLBI detections be due to spatial substructure within the general ISM starburst-powered radio emission? As noted in Section 4.5 the flux densities on the $\mathrm{Eb}-\mathrm{Wb}$ and $\mathrm{Eb}-\mathrm{Jb} 1$ baselines are usually comparable thus implying a source size less than about half of the $\mathrm{Eb}-\mathrm{Jb} 1$ fringe spacing, i.e., $\lesssim 10$ mas. A typical VLBI detection flux density of $\sim 3 \mathrm{mJy}$ would then imply a brightness temperature of at least $10^{6.2} \mathrm{~K}$. Even larger brightness temperatures are found on the Eb-Ar baseline where sub-milliarcsecond sizes imply brightness temperatures of $\gtrsim 10^{8} \mathrm{~K}$. These high brightness temperatures are inconsistent with star formation-powered synchrotron emission which is predicted to have brightness temperatures of $\lesssim 10^{5} \mathrm{~K}$ (Condon et al. 1991).

Given that we only have fringe detections on single baselines it is possible that such detections (especially on the Eb-Jb1 baseline) could be due to star formation-powered radio emission combined with an unusual source brightness distribution. One such distribution would be a thin rectangle with its small dimension $<10$ mas along the direction of the fringe spacing; this would however require both unusual brightness distributions and chance alignments in position angle in a number of sources to affect our results. A second such distribution would be one with order $100 \%$ amplitude fluctuations of $10^{5} \mathrm{~K}$ brightness on scales $<10$ mas over a region of order 1000 mas in diameter, giving large rms visibilities of the same size on $\mathrm{Eb}-\mathrm{Wb}$ and $\mathrm{Eb}-\mathrm{Jb} 1$. The ratio of detected visibilities on these two baselines seems too close to unity to be explained by such a random model. Additionally, such a brightness distribution seems very unlikely, one would instead expect larger fluctuations as we go to larger separations within the source giving rise to larger rms visibilities on the shorter baseline. This is what is in fact observed in Arp 220. Taking the data of Parra et al. (2007) and removing the effect of the compact sources we
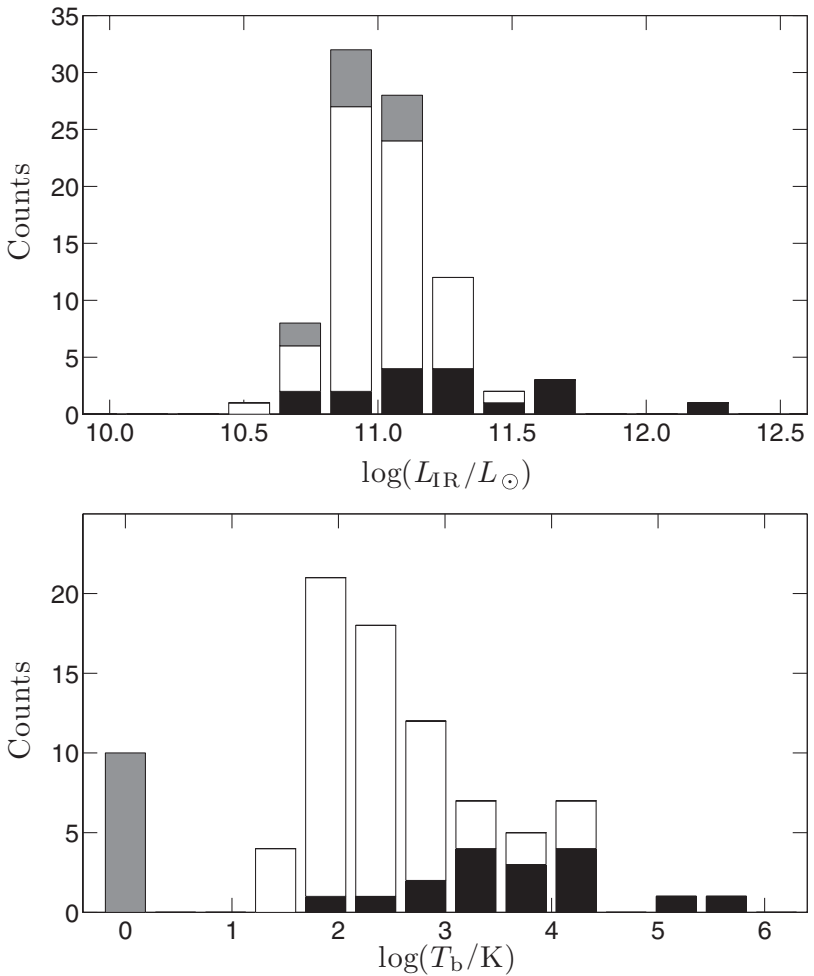

Figure 5. Histograms of IR luminosity (top panel) and peak brightness temperature of VLA scale radio component (bottom panel) for VLBI observed sources. In both panels VLBI detections are shown in black. VLBI nondetections for sources respectively detected and undetected by the VLA are shown in white and gray, respectively. The brightness temperature in the bottom panel is calculated after subtraction of the VLBI component from the VLA map (see Section 4.2). Note for ease of comparison the three VLBI coredominated detections (i.e 00005+2140,03164+4119, 06239+7428) are not plotted in either panel. A much stronger correlation between VLBI detection and high brightness temperature VLA scale emission is found than with IR luminosity (see Section 4.5 and also Figure 8 )

detect the diffuse emission on $\mathrm{Eb}-\mathrm{Wb}$ but not on the $\mathrm{Eb}-\mathrm{Jb} 1$ baseline. Since Arp 220 is brighter and more compact than other COLA sources it seems unlikely that the Eb-Jb1 detections in these can be due to randomly fluctuating diffuse $10^{5} \mathrm{~K}$ emission.

\subsubsection{Compact Sources in Arp 220}

For the case of Arp $220(15327+2340)$, most of the flux density detected in our VLBI snapshot observations clearly comes from non-AGN activity. This galaxy has been known for many years (Smith et al. 1998; Lonsdale et al. 2006; Parra et al. 2007) to harbor multiple compact radio sources consistent with a mixed population of luminous radio SNRs (interacting with the ISM) and $\mathrm{SNe}$ (interacting with progenitor winds). Arp 220 is however a very unusual source within the COLA sample being by far its most IR luminous object. It follows that a confirmed SNR/SNe origin for the VLBI snapshot fringes in Arp 220 does not necessarily imply that the same explanation is valid for the rest of the VLBI detections. The diagonal line in Figure 4 shows the Eb-Wb spectral luminosity of Arp 220 scaled linearly with $L_{\mathrm{IR}}$ and demonstrates that if the VLBI contribution from SNR/ $\mathrm{SNe}$ scales linearly with star formation rate then these cannot explain the VLBI detections. In the next subsections, we discuss in more detail whether SNRs and $\mathrm{SNe}$ can in fact explain the VLBI detections. 


\subsubsection{Supernova Remnant Origin}

Chomiuk \& Wilcots (2009) find in their study of the radio luminosity function of SNRs in nearby normal and starburst galaxies a constant power-law exponent and a scaling factor proportional to SFR. They also find both theoretically and empirically that the luminosity of the brightest SNR in each galaxy scales linearly with the SFR. Since even on our shortest VLBI baseline bright SNRs will be separated by several fringe spacings their expected contribution to the correlated VLBI flux density will be an incoherent sum of all the SNR flux densities. This incoherent sum will, however, because of the constant slope SNR luminosity function found by Chomiuk \& Wilcots (2009) simply be some multiple of the brightest SNR flux density. Since this latter quantity is linearly correlated with SFR we expect the total observed VLBI signal due to SNRs to also linearly scale with SFR. Since about half of the flux density seen on the Eb-Wb baseline for Arp 220 comes from SNR a VLBI contribution which lies a factor of two below the diagonal line shown in Figure 4 is therefore expected; in all cases this lies below the luminosity of the detected VLBI cores.

In fact estimating the SNR contribution to VLBI flux density in COLA galaxies by scaling Arp 220 by $L_{\mathrm{IR}}$ as described above probably results in an overestimate of the SNR contribution for COLA galaxies. This is because Chomiuk \& Wilcots (2009) find that although the SNR luminosity function in Arp 220 has the same slope as in other galaxies the scaling factor between SNR brightness and SFR is larger (possibly because of the very high gas density in Arp220). The true VLBI SNR contribution in COLA galaxies although having the same linear slope as drawn in Figure 4 therefore likely passes below the Arp 220 point; because this source is over-luminous in SNR for its SFR.

\subsubsection{Radio Supernova Origin}

A difficulty for calculating the possible contribution of radio SNe to our detected VLBI flux density is that unlike the case for radio SNR no well defined radio SNe luminosity functions have been published. It is clear however that only the most luminous Type Ic or Type IIn optical classes (Chevalier 2006) would be detectable at the distance of COLA galaxies. The most powerful Type Ic objects are often associated with $\gamma$-ray bursts and have rapid rise and decay times. The slightly weaker Type IIn $\mathrm{SNe}$ are much longer lived and hence more likely to be detected. Optically, about $4 \%$ of core-collapse SNe are of Type IIn (Smartt et al. 2009), however even these show a wide range in radio luminosity. For instance all of the six IIn's observed by van Dyk et al. (1996) were undetected in the radio with $4.8 \mathrm{GHz}$ spectral luminosity $<1.4 \times 10^{19} \mathrm{~W} \mathrm{~Hz}^{-1}$. The likely Type IIn's in Arp 220 (Parra et al. 2007) have spectral luminosity of $\sim 4 \times$ $10^{20} \mathrm{~W} \mathrm{~Hz}^{-1}$. The most radio luminous Type IIn so far detected $\left(2 \times 10^{21} \mathrm{~W} \mathrm{~Hz}^{-1}\right.$ at $\left.4.8 \mathrm{GHz}\right)$ is SN1986J in NGC 891 (Rupen et al. 1987). Sources close to this maximum seem however to be rare. Considering the number of optically identified Type IIn's searched in the radio (see Chandra et al. 2009, and references therein) in normal galactic disks we can estimate that at most one in ten Type IIn's (or $0.4 \%$ of all core collapse $\mathrm{SNe}$ ) reaches to within a factor of two of the SN1986J radio luminosity.

Among COLA VLBI detections there are six objects other than Arp 220 with luminosities above that of SN1986J at maximum light (i.e., above the dashed line in Figure 4). A single $\mathrm{RSNe}$ origin seems to be ruled out for these objects and we are forced to consider models consisting of two or more extremely luminous objects. Such SN1986J-like objects stay within a factor of two of their peak $4.8 \mathrm{GHz}$ luminosity for $\sim 5$ years and host galaxies of these VLBI detections have total SN rates of order 0.5 year $^{-1}$. If $0.4 \%$ of core collapse are SN1986J-like, then we expect 0.01 such objects on average per COLA galaxy. The probability of getting just one example of two or more such luminous objects in a galaxy within our whole sample is thus only $1.5 \%$.

Figure 4 shows 7 VLBI detections at $\sim 1 \times 10^{20} \mathrm{~W} \mathrm{~Hz}^{-1}$ that could potentially be explained as single SN1986J-like objects observed when above $50 \%$ of maximum light. Assuming the same radio luminous $\mathrm{SNe}$ lifetimes and fractions as before and typical SFRs of this group of $\sim 0.25 \mathrm{yr}^{-1}$ there should only be 0.5 such objects in the whole sample. Finally there are three VLBI detections at $\sim 0.6 \times 10^{20} \mathrm{~W} \mathrm{~Hz}^{-1}$, it is possible that one of these objects could be a $\mathrm{SNe}$, though without a detailed radio $\mathrm{SN}$ luminosity function it is hard to make any definite statements.

A general argument that suggests that the vast majority of our VLBI detections are not explained by SNe comes from the fact (see Section 4.3) that all our VLBI detections are well above our detection limit. If the origin of the VLBI detections were single or multiple RSNe such a gap in luminosity between our weakest detection and the sensitivity limit is hard to explain. A single RSN spends a much longer period at low luminosities than at high, and intrinsically weaker $\mathrm{SNe}$ are more common than strong ones, so one would expect many more detections just above the detection cutoff than are observed.

We conclude that, while one or two of the less luminous detections could be caused by radio $\mathrm{SNe}$, the vast majority of our VLBI detections cannot and must instead be AGN powered.

\subsection{Nature of Extended Radio Emission}

For most VLBI detected COLA sources $(17 / 20)$, a large fraction of the total radio flux density arises from several $100 \mathrm{pc}$ scale structures seen in the VLA maps. As noted in Section 4.1 the total flux densities of nearly all COLA sources, including both VLBI detections and non-detections (see Section 4.4 and Figure 1), closely follows the FIR to radio correlation at both 1.4 and $4.8 \mathrm{GHz}$. This result indicates that most of the radio emission recovered in the VLA maps is powered by star formation activity. It is known from observations of the starbursts in M82 and Arp 220 that the associated star formation induced compact emission from SNR and SNe is just a few percent (Lonsdale et al. 2006) of the total diffuse component radio emission; hence there is no contradiction between star formation being the main power source for the total radio emission and our conclusion that it does not provide enough correlated flux density to explain the VLBI detections.

\subsection{Nature of integrated IR Emission}

Figure 7 shows a IR color-color diagram for the 90 sources with VLBI observations in the COLA North sample. Any position on this diagram can be reached by a linear combination of starburst temperature, AGN and reddening (Kewley et al. 2000). It is therefore impossible to unambiguously estimate the proportions in which these ingredients are combined. However, most of the sources in the sample appear to follow the line traced by blackbodies of progressively increasing temperatures (i.e., the starburst line) with a scatter apparently increasing with temperature suggesting a larger AGN contribution to the IR energy budget in warm starbursts. The overall scatter of the COLA sample is however much smaller than that observed by 


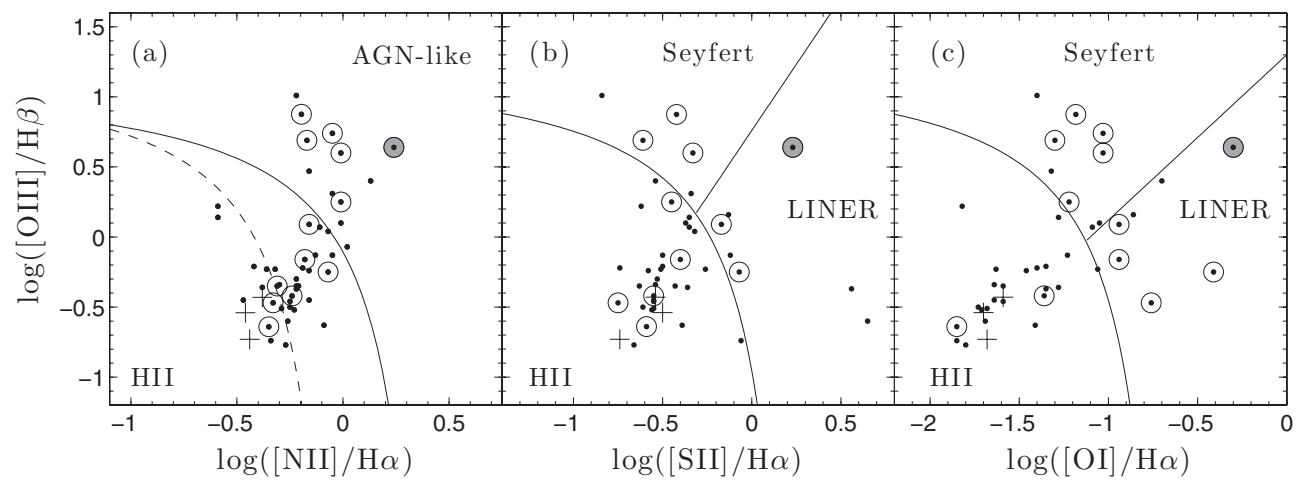

Figure 6. Optical-line diagnostic diagrams ("BPT" diagrams) for the COLA-N sources that are both VLBI observed and have available optical line data. Dots indicate VLA detected sources and crosses those undetected by the VLA. VLBI detected sources are shown circled. (a) $[\mathrm{N} \mathrm{II}] / \mathrm{H} \alpha$ vs. [O III]/H $\beta$ diagram. The solid and dashed curves are respectively the theoretical Kewley et al. (2001) extreme starburst line and the Kauffmann et al. (2003) empirical line for star-forming galaxies. Sources that lie between the two curves are classified as composite Starburst/AGN. (b) $[\mathrm{SII}] / \mathrm{H} \alpha$ vs. [O III]/H $\beta$ diagram. (c) $[\mathrm{OI}] / \mathrm{H} \alpha$ vs. $[\mathrm{O}$ III]/H $\beta$ diagram. In the latter two panels, the solid curved line is the extreme starburst line from Kewley et al. (2001) and the straight diagonal line separates Seyferts from LINERS (Kewley et al. 2006). Note that for some sources not all line ratios are plotted because some lines were not detected. The shaded VLBI detected source is $15327+2340$ (Arp220).

Rush et al. (1993) in their sample of optically selected Seyferts. We therefore argue that for most sources in our sample that their IR emission is mostly powered by starburst activity with any AGN contribution being energetically unimportant.

All 10 VLA non-detections (see Section 4.2) with VLBI data are clustered near the cold end of the starburst line (crosses in Figure 7). As discussed in Section 4.2 these are likely to be large sources whose relatively large FIR luminosity is not due to concentrated nuclear starburst activity but is instead caused by the aggregation of normal star forming regions spread over their large galactic disks.

\subsection{Comparison of Optical and Radio Source Classifications}

As discussed in Section 4.6, we find that the fraction of VLBI detections in AGN-like optical types is substantially higher than in H II type galaxies, supporting the interpretation that VLBI detections indicate AGN activity. We find however no one-toone correspondence between the optical classification and VLBI detection. The fact that half of the observed Seyfert sources (4/ 8 ) were not detected by VLBI supports the result of Corbett et al. (2003) who found observationally two classes of Seyferts with and without detectable VLBI radio cores. This could simply be an artifact of our limited VLBI sensitivity or it could mean that there are physically two classes of Seyfert with different degrees of radio-loudness. The relatively high VLBI detection rate of LINER sources $(4 / 6)$ is consistent with models in which this class is usually AGN powered (Kewley et al. 2006) although it is clear that in some sources LINER-like spectra can arise in shocks, i.e., for the case of Arp 220 (Arribas et al. 2001). The relatively low incidence of VLBI detections in $\mathrm{H}$ II galaxies (2/ 15) suggests that cases of completely optically obscured AGN are rare. Combining the VLBI and optical results we find the overall incidence of AGN activity to be high. If we add the four non-VLBI detected Seyferts to the 19 VLBI detections (excluding Arp 220) we get a minimum fraction of sources containing AGN of 22/90 (25\%).

\subsection{Implications for the AGN Starburst Connection}

An interesting correlation found in the present work is that between the presence of a VLBI detection and of high brightness arcsecond-scale radio emission (see Section 4.5 and Figure 5). In Section 5.1 we argued that VLBI detections are due to AGN

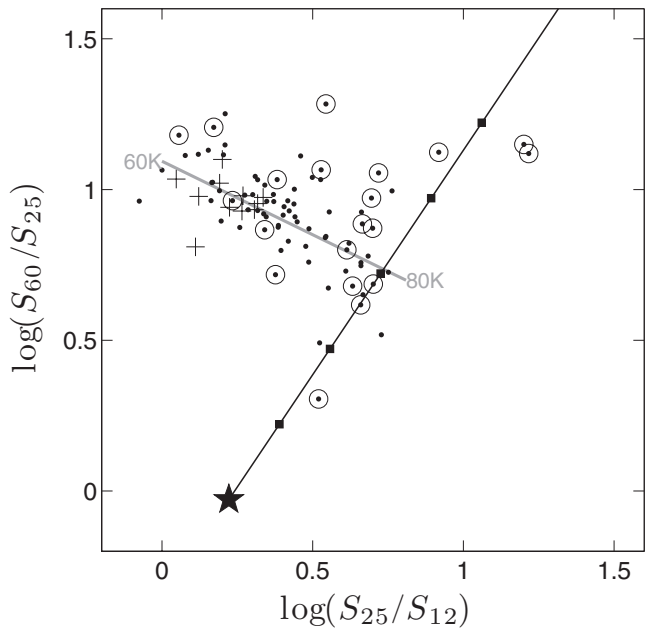

Figure 7. IR color-color diagram for COLA North sources observed by VLBI. VLBI detections are indicated with open circles and VLA non detections as crosses. The gray line is traced by blackbodies of temperatures progressing from 60 to $80 \mathrm{~K}$ from the left. The black line is a reddening line starting from the average warm Seyfert 1 nucleus (indicated by a star). Squares over this line are located at $\tau_{25} \mu \mathrm{m}=1,2,3,4$, and 5 (see Dopita et al. 1998).

activity rather than SNe or SNRs. In Section 5.2, we further argued that the extended radio emission seen with the VLA is due to star formation. Taken together these results imply a strong connection between AGN activity and the presence of a compact starburst.

A significant body of evidence in favor of a strong starburst/ AGN connection has been accumulating in recent years. In nearby AGNs evidence is seen for for nuclear post-starburst stellar populations of age $\sim 10^{6}-10^{8}$ year (Davies et al. 2007) suggesting a possible evolutionary connection with nuclear starbursts preceding AGN activity (see also Heckman 2008). There are however also many individual examples of concurrent starburst/AGN activity (e.g., Fathi et al. 2006). The present paper has found evidence linking AGN activity with concurrent compact starbursts in FIR selected sources and it is interesting to consider possible physical interpretations of this result.

The Eddington-limited starbursts discussed by Thompson et al. (2005), which can also feed central AGN, are predicted to have central peak bolometric brightness of $10^{13} L_{\odot} \mathrm{kpc}^{-2}$ 


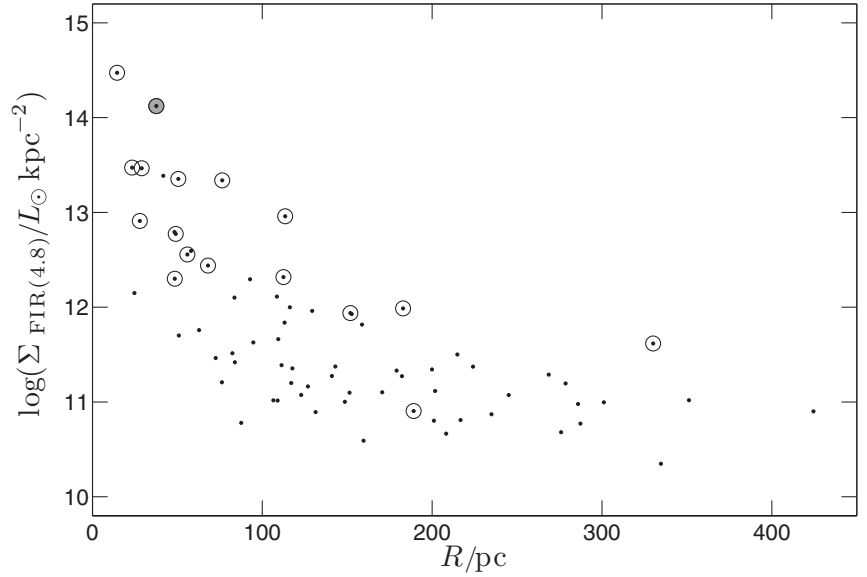

Figure 8. Starburst FIR equivalent brightness $\Sigma_{\mathrm{FIR}(4.8)}$ vs. central starburst component radius $R$ for sources both imaged by the VLA and observed by VLBI. VLBI detections are indicated by a circle with Arp 220 emphasized as a filled circle. This diagram duplicates a similar one in Thompson et al. (2005) for more luminous IR galaxies. In this plot $\Sigma_{\mathrm{FIR}(4.8)}=L_{\mathrm{FIR}(4.8)} / A_{\text {radio }}$ where $L_{\mathrm{FIR}(4.8)}$ is the equivalent starburst FIR spectral luminosity estimated from the $4.8 \mathrm{GHz}$ data and $A_{\text {radio }}$ is the area of the central starburst source. $L_{\mathrm{FIR}(4.8)}$ is calculated by transforming the $4.8 \mathrm{GHz}$ spectral luminosity of the brightest VLA component $L_{4.8 \text { peak }}$ to an equivalent FIR spectral luminosity using the FIR-radio correlation.

consistent with the luminous compact starbursts from Condon et al. (1991). Figure 8 plots the estimated FIR surface brightness versus fitted size for the arcsecond-scale radio structures for our COLA sources, matching Figure 4 of Thompson et al. (2005). We find that our VLBI sources are mostly detected in sources with bolometric brightness approaching or around $10^{13} L_{\odot} \mathrm{kpc}^{-2}$ which coincides with values predicted for Eddington-limited starbursts.

Thompson et al. (2005) also argued for two separate modes by which such compact starbursts can feed a central black hole, supplying either $0.1 M_{\odot}$ year $^{-1}$ or $4 M_{\odot}$ year $^{-1}$ depending on whether the gas supply rate at several hundred parsec radius is smaller or larger than $220 M_{\odot} \mathrm{yr}^{-1}$. The rest of this mass is converted into stars. Since the star formation rates of COLA sources are $\ll 220 M_{\odot} \mathrm{yr}^{-1}$ the lower black hole supply rate applies. Assuming an efficiency of $10 \%$ this supply rate translates into an AGN bolometric luminosity of $1.5 \times$ $10^{11} L_{\odot}$ which is comparable to the IR luminosities of the COLA galaxies with VLBI detections. Such a high AGN luminosity, which would mostly emerge in the IR, is inconsistent with the fact that COLA sources lie on the FIR-radio correlation and have IR colors typically found in starburst-powered sources (see 5.3). We can speculate that a constant fraction of gas supplied to the black hole might be lost to winds or jets formed close to the black hole event horizon, thus reducing the AGN bolometric luminosity. The detected core radio emission would be generated at the base of these jets (Falcke \& Biermann 1995). The starburst regulation of the gas supply would ensure that these jets had similar mass outflow rates and mechanical luminosities therefore explaining the relatively narrow range of radio luminosity of our VLBI detections.

The high incidence we find of AGN activity in compact high brightness starbursts (i.e., VLBI detections in 13/18 sources with specific star formation rates $>10^{12} L_{\odot} \mathrm{kpc}^{-2}$, see Figure 8) suggests only a short interval between the triggering of such starbursts and the onset of AGN activity. In contrast Davies et al. (2007) argue (based on AGN stellar population studies) that there is a 50-100 Myr delay between the peak of nuclear starburst activity and the onset of AGN activity. These studies were however done on AGN selected sources. AGNs plausibly last much longer after the initial feeding event than does starburst activity. Possibly, this is because once the central $\sim 1$ pc scale accretion disk is fueled with gas via a compact starburst long viscous accretion times (up to $10^{9}$ yr. King \& Pringle 2007) allow AGN activity to continue long after starburst activity has faded. Given this timescale difference examples of currently active nuclear starbursts in AGN selected samples might well be expected to be rare. Additionally, during the initial composite AGN/starburst phase optical obscuration may be very large making such objects hard to be optically identified as AGN. Since COLA is FIR selected and we have used radio observations as the primary means to detect AGN both of the above biases are avoided allowing the detection of the initial phases of composite AGN/starburst activity.

Both our own observations and those of Condon et al. (1991) show a strong correlation between high FIR luminosity and compact high brightness starbursts. Within the IRAS-BGS sample Condon et al. (1991) found that all starbursts with $L_{\text {FIR }}>10^{11.8} L_{\odot}$ were in fact of this high brightness Eddingtonlimited type. If the correlation between AGN powered VLBI sources and high brightness shown in Figure 8 also holds for this more luminous sample then one would expect all of these to have AGN activity detectable via VLBI. It can be argued that Arp 220 is a clear counter-example since it has high radio brightness and although detected by VLBI this detection is due to $\mathrm{SNe}$ and SNRs rather than an AGN core or lobes. However, as noted by Parra et al. (2007) within the western nucleus of Arp 220 there exist several candidate sources for a radio AGN. Recent millimeter continuum and ${ }^{12} \mathrm{CO}(2-1)$ spectral line interferometry results by Downes \& Eckart (2007) and Sakamoto et al. (2008) strongly bolster the case that a powerful AGN does in fact exist in the western nucleus of Arp 220.

More generally, within snapshot $1.4 \mathrm{GHz}$ VLBI observations conducted by Lonsdale et al. (1993), about $50 \%$ of luminous IRAS-BGS sources were detected on global baselines. These detections had however ambiguous interpretations being explainable by both AGN and radio $\mathrm{SNe} / \mathrm{SNR}$ models. In general, at other wavebands attempts to definitely detect or reject AGN contributions in these very luminous starbursts have also often proved inconclusive. If as predicted by the Thompson et al. (2005) model for Eddington-limited starbursts the gas supply to the central black hole is relatively constant for compact starburst sources we may obtain the same AGN luminosity regardless of $L_{\mathrm{IR}}$. It follows that in more intense higher IR luminosity starbursts it may be harder to detect the AGN component in the midst of all the starburst activity. Paradoxically it may be that within the moderate luminosity COLA sample a strong correlation between intense starburst activity and radio AGNs is easier to detect than in more luminous samples. This is firstly because COLA straddles a luminosity range were there are large numbers of both low brightness and Eddington-limited starbursts. Second, $L_{\mathrm{IR}}$ is not high enough (except for Arp 220) that there is significant confusion in the radio between AGN and starburst induced structure.

It is clear that both the VLBI snapshot observations of COLA-N presented in this paper and the observations of IRASBGS sources carried out by Lonsdale et al. (1993) suffer from lack of uv coverage. New long track VLBI observation which produce good quality images would help distinguish star 
formation origins of VLBI flux density (by for instance seeing multiple compact VLBI components indicating star formation, e.g., Parra et al. 2007) from AGN origins (core-jet morphology sources). Observations of half of the VLBI detected COLA$\mathrm{N}$ sources have already been made using the EVN at $6 \mathrm{~cm}$ wavelength. These data are presently being reduced.

\section{CONCLUSIONS}

The main conclusions of this paper are the following.

1. We have made $4.8 \mathrm{GHz}$ VLA images for 95 of the 110 sources in the COLA North sample. Of these sources, 28, 62, and 5 sources have respectively Core, Core+Extended, and Extended radio morphologies as defined by Neff \& Hutchings (1992). The 15 non-detected sources are likely extended (resolved out) galaxies with radio surface brightnesses below our sensitivity (see Section 4.2).

2. We argue that the properties of the $4.8 \mathrm{GHz}$ radio emission structures imaged on scales of a few $100 \mathrm{pc}$ are consistent with being powered by star formation activity because the recovered flux density in these structures follows the FIR to radio correlation for star forming galaxies (see Section 4.1).

3. We detect $22 \%(20 / 90)$ of the sources observed in the VLBI snapshots (see Section 4.3). Assuming that these VLBI detections are single Gaussian components the simultaneous detection at multiple baselines in most cases constrains the size of the emitting region to parsec scales.

4. The majority of the VLBI detections are well above our detection threshold (see Figure 4) suggesting a lower limit of $\sim 10^{21} \mathrm{~W} \mathrm{~Hz}^{-1}$ for the luminosity of these compact sources.

5. Based on luminosity arguments we can rule out a radio SNR origin for all of the VLBI detections (see Section 5.1.3).

6. We can also rule out a radio SN origin for most VLBI detections unless we assume that the incidence of powerful radio supernovae is much larger in COLA sources than in most galactic disks. We estimate that at most one or two of the VLBI detections could be due to radio SN.

7. Our VLBI detections are preferentially found in sources with the highest radio brightness arcsecond-scale radio emission, even after subtracting off the VLBI cores. Since this arcsecond-scale radio emission is powered by star formation activity (see point 2 above) we conclude that there is a relationship between compact starbursts and AGN activity. This is consistent with the proposal of Thompson et al. (2005) that Eddington-limited nuclear starbursts can feed AGN.

8. The high AGN incidence among compact starbursts implies no significant delay between the period of intense nuclear starburst activity and the onset of AGN activity. If the AGN activity continues long after the starburst has faded this would explain the predominance of post starburst stellar populations in AGN sources.

We thank the anonymous referee for his/her very helpful comments on an earlier version of this paper. R.P. acknowledges a Chalmers University PhD student stipend and FONDECYT postdoctoral research grant 3085032. J.C. acknowledges a Swedish VR grant. The European VLBI Network is a joint facility of European, Chinese, South African and other radio astronomy institutes funded by their national research councils. The Arecibo Observatory is the principal facility of the National Astronomy and Ionosphere Center which is operated by Cornell University under a cooperative agreement with the National Science Foundation. The National Radio Astronomy Observatory is a facility of the National Science Foundation operated under a cooperative agreement by Associated Universities, Inc. This research has made use of the NASA/IPAC Extragalactic Database (NED) which is operated by the Jet Propulsion Laboratory, California Institute of Technology, under contract with the National Aeronautics and Space Administration.

\section{REFERENCES}

Arribas, S., Colina, L., \& Clements, D. 2001, ApJ, 560, 160

Baldwin, J. A., Phillips, M. M., \& Terlevich, R. 1981, PASP, 93, 5

Beichman, C. A., Neugebauer, G., Habing, H. J., Clegg, P. E., \& Chester, T. J., (ed.) 1988, IRAS Catalogs and Atlases., Vol. 1: Explanatory Supplement (Pasadena, CA: JPL)

Chandra, P., et al. 2009, ApJ, 690, 1839

Chevalier, R. A. 2006, arXiv:astro-ph/0607422

Chomiuk, L., \& Wilcots, E. M. 2009, ApJ, 703, 370

Condon, J. J. 1992, ARA\&A, 30, 575

Condon, J. J., Cotton, W. D., Greisen, E. W., Yin, Q. F., Perley, R. A., Taylor, G. B., \& Broderick, J. J. 1998, AJ, 115, 1693

Condon, J. J., Huang, Z.-P., Yin, Q. F., \& Thuan, T. X. 1991, ApJ, 378, 65

Corbett, E. A., et al. 2002, ApJ, 564, 650

Corbett, E. A., et al. 2003, ApJ, 583, 670

Davies, R. I., Sánchez, F. M., Genzel, R., Tacconi, L. J., Hicks, E. K. S., Friedrich, S., \& Sternberg, A. 2007, ApJ, 671, 1388

Dopita, M. A., Heisler, C., Lumsden, S., \& Bailey, J. 1998, ApJ, 498, 570

Downes, D., \& Eckart, A. 2007, A\&A, 468, L57

Falcke, H., \& Biermann, P. L. 1995, A\&A, 293, 665

Fathi, K., Storchi-Bergmann, T., Riffel, R. A., Winge, C., Axon, D. J., Robinson, A., Capetti, A., \& Marconi, A. 2006, ApJ, 641, L25

Häring, N., \& Rix, H.-W. 2004, ApJ, 604, L89

Heckman, T. M. 2008, arXiv:0809.1101

Helou, G., Soifer, B. T., \& Rowan-Robinson, M. 1985, ApJ, 298, L7

Hinshaw, G., et al. 2009, ApJS, 180, 225

Kauffmann, G., et al. 2003, MNRAS, 346, 1055

Kellermann, K. I., Fomalont, E. B., Mainieri, V., Padovani, P., Rosati, P., Shaver, P., Tozzi, P., \& Miller, N. 2008, ApJS, 179, 71

Kewley, L. J., Dopita, M. A., Sutherland, R. S., Heisler, C. A., \& Trevena, J. 2001, ApJ, 556, 121

Kewley, L. J., Groves, B., Kauffmann, G., \& Heckman, T. 2006, MNRAS, 372, 961

Kewley, L. J., Heisler, C. A., Dopita, M. A., Sutherland, R., Norris, R. P., Reynolds, J., \& Lumsden, S. 2000, ApJ, 530, 704

King, A. R., \& Pringle, J. E. 2007, MNRAS, 377, L25

Knapen, J. 2004, in ASP Conf. Ser. 320, The Neutral ISM in Starburst Galaxies, ed. S. Aalto, S. Huttemeister, \& A. Pedlar (San Francisco, CA: ASP), 205

Lonsdale, C. J., Diamond, P. J., Thrall, H., Smith, H. E., \& Lonsdale, C. J. 2006, ApJ, 647, 185

Lonsdale, C. J., Smith, H. J., \& Lonsdale, C. J. 1993, ApJ, 405, L9

Magorrian, J., et al. 1998, AJ, 115, 2285

Merloni, A., Heinz, S., \& di Matteo, T. 2003, MNRAS, 345, 1057

Mould, J. R., et al. 2000, ApJ, 529, 786

Neff, S. G., \& Hutchings, J. B. 1992, AJ, 103, 1746

Norris, R. P., et al. 2006, AJ, 132, 2409

Parra, R. 2007, PhD thesis, Chalmers Tekniska Hogskola (Sweden)

Parra, R., Conway, J. E., Diamond, P. J., Thrall, H., Lonsdale, C. J., Lonsdale, C. J., \& Smith, H. E. 2007, ApJ, 659, 314

Pearson, T. J. 1999, in ASP Conf. Ser. 180, Synthesis Imaging in Radio Astronomy II, ed. G. B. Taylor, C. L. Carilli, \& R. A. Perley (San Francisco, CA: ASP), 335

Prandoni, I., Gregorini, L., Parma, P., de Ruiter, H. R., Vettolani, G., Wieringa, M. H., \& Ekers, R. D. 2000, A\&AS, 146, 41

Prieto, M. A., Maciejewski, W., \& Reunanen, J. 2005, AJ, 130, 1472

Prieto, M. A., Maciejewski, W., \& Reunanen, J. 2007, AJ, 133, 1847

Rovilos, E., Diamond, P. J., Lonsdale, C. J., Lonsdale, C. J., \& Smith, H. E. 2003, MNRAS, 342, 373

Rupen, M. P., van Gorkom, J. H., Knapp, G. R., Gunn, J. E., \& Schneider, D. P. 1987, AJ, 94, 61

Rush, B., Malkan, M. A., \& Spinoglio, L. 1993, ApJS, 89, 1

Sakamoto, K., et al. 2008, ApJ, 684, 957

Sanders, D. B., Mazzarella, J. M., Kim, D.-C., Surace, J. A., \& Soifer, B. T. 2003, AJ, 126, 1607

Sanders, D. B., \& Mirabel, I. F. 1996, ARA\&A, 34, 749 
Schartmann, M., Burkert, A., Krause, M., Camenzind, M., Meisenheimer, K., \& Davies, R. I. 2010, MNRAS, 219

Smartt, S. J., Eldridge, J. J., Crockett, R. M., \& Maund, J. R. 2009, MNRAS, 395, 1409

Smith, H. E., Lonsdale, C. J., \& Lonsdale, C. J. 1998, ApJ, 492, 137

Thompson, T. A., Quataert, E., \& Murray, N. 2005, ApJ, 630, 167

van Buren, D., \& Greenhouse, M. A. 1994, ApJ, 431, 640 van Dyk, S. D., Weiler, K. W., Sramek, R. A., Schlegel, E. M., Filippenko, A. V., Panagia, N., \& Leibundgut, B. 1996, AJ, 111, 1271

Veilleux, S., \& Osterbrock, D. E. 1987, ApJS, 63, 295

Wunderlich, E., Wielebinski, R., \& Klein, U. 1987, A\&AS, 69, 487

Yuan, T., Kewley, L. J., \& Sanders, D. B. 2010, ApJ, 709, 884

Yun, M. S., Reddy, N. A., \& Condon, J. J. 2001, ApJ, 554, 803

Zamfir, S., Sulentic, J. W., \& Marziani, P. 2008, MNRAS, 387, 856 